\title{
High dose saccharin supplementation does not induce gut microbiota dysbiosis or glucose intolerance in healthy humans and mice
}

Joan Serrano

Ohio State University

Kathleen R Smith

Ohio State University

Audra L Crouch

Ohio State University

Vandana Sharma

Sanford Burnham Prebys Medical Discovery Institute

Fanchao Yi

AdventHealth

Veronika Vargova

AdventHealth

Traci E LaMoia

Ohio State University

Lydia M Dupont

Ohio State University

Vanida Serna

Ohio State University

Fenfen Tang

Ohio State University

Laisa Gomes-Dias

Ohio State University

Joshua Blakeslee

Ohio State University

Emmanuel Hatzakis

Ohio State University

\section{Scott N Peterson}

Sanford Burnham Prebys Medical Discovery Institute

Matthew Anderson

Ohio State University

Richard E Pratley 
AdventHealth

George Kyriazis ( $\sim$ kyriazis.2@osu.edu )

The Ohio State University Wexner Medical Center https://orcid.org/0000-0001-9514-3482

\section{Research}

Keywords: artificial sweeteners; saccharin; sweet taste receptors; gut microbiota; glucose intolerance; short-chain fatty acids; fecal metabolomics; T1R2; dysbiosis

Posted Date: February 14th, 2020

DOl: https://doi.org/10.21203/rs.2.23608/v1

License: (c) (i) This work is licensed under a Creative Commons Attribution 4.0 International License. Read Full License 
1 High dose saccharin supplementation does not induce gut microbiota dysbiosis or

2 glucose intolerance in healthy humans and mice

3 Author list:

41 Joan Serrano. Department of Biological Chemistry \& Pharmacology, College of Medicine, The

5 Ohio State University, Columbus, OH, USA. joan.serrano@osumc.edu

62 Kathleen R. Smith. Department of Biological Chemistry \& Pharmacology, College of Medicine,

7 The Ohio State University, Columbus, OH, USA. Kathleen.smith@pfizer.com

83 Audra L Crouch. Department of Microbiology, College of Arts \& Sciences, The Ohio State

$9 \quad$ University, Columbus, OH, USA. crouch.115@buckeyemail.osu.edu

104 Vandana Sharma. Sanford Burnham Prebys Medical Discovery Institute, La Jolla, CA, USA.

11 vsharma@sbpdiscovery.org

125 Fanchao Yi. Translational Research Institute for Metabolism and Diabetes, Advent-Health,

13 Orlando FL, USA. Fanchao.Yi@AdventHealth.com

146 Veronika Vargova. Translational Research Institute for Metabolism and Diabetes, Advent-

15 Health, Orlando FL, USA. v.cigerova@gmail.com

167 Traci E. LaMoia. Department of Biological Chemistry \& Pharmacology, College of Medicine,

17 The Ohio State University, Columbus, OH, USA. traci.lamoia@yale.edu

188 Lydia M. Dupont. Department of Biological Chemistry \& Pharmacology, College of Medicine,

19 The Ohio State University, Columbus, OH, USA. Dupont.41@osu.edu

209 Vanida Serna. Department of Biological Chemistry \& Pharmacology, College of Medicine, The

21 Ohio State University, Columbus, OH, USA. Vanida.Serna@osumc.edu

2210 Fenfen Tang. Department of Food Science and Technology, College of Food, Agricultural \&

23 Environmental Sciences, The Ohio State University, Columbus, OH, USA.

24 tang.1263@buckeyemail.osu.edu 
11 Laisa Gomes-Dias. Department of Horticulture and Crop Science, College of Food, Agricultural \& Environmental Sciences, The Ohio State University, Columbus, OH, USA.

27 gomesdias.1@osu.edu

12 Joshua Blakeslee. Department of Horticulture and Crop Science, College of Food, Agricultural

\& Environmental Sciences, The Ohio State University, Columbus, $\mathrm{OH}$, USA. blakeslee.19@osu.edu

13 Emmanuel Hatzakis. Department of Food Science and Technology, College of Food,

32 Agricultural \& Environmental Sciences, The Ohio State University, Columbus, OH, USA.

33 chatzakis.1@osu.edu

3414 Scott N. Peterson. Sanford Burnham Prebys Medical Discovery Institute, La Jolla, CA, USA.

35 speterson@sbpdiscovery.org

15 Matthew Anderson. Department of Microbiology, College of Arts \& Sciences, The Ohio State

37 University, Columbus, OH, USA. anderson.3196@osu.edu

16 Richard E. Pratley. Translational Research Institute for Metabolism and Diabetes, AdventHealth, Orlando FL, USA. Richard.Pratley.MD@AdventHealth.com

17 Corresponding author: George A. Kyriazis. Department of Biological Chemistry \&

41 Pharmacology, College of Medicine, The Ohio State University, Columbus, OH, USA.

42 Georgios.Kyriazis@osumc.edu

\section{Abstract}

44 Background: Non-caloric artificial sweeteners (NCAS) are widely used as a substitute for dietary 45 sugars to control body weight or glycemia. Paradoxically, saccharin and other NCAS have been

46 reported to induce glucose intolerance in mice fed a high-fat diet and in a subset of humans by 47 directly inducing unfavorable changes in gut microbiota. These findings have raised concerns 48 about NCAS and called into question their broad use. Whether these results can be generalized 49 to healthy populations consuming conventional diets is unknown. It is also unclear how different 50 NCAS, that do not share a common chemical structure, can produce identical direct effects on 
51 gut microbiota. A common feature of all NCAS is their strong affinity for sweet taste receptors

52 (STRs) which are expressed in the intestine. However, their role in mediating NCAS-induced 53 effects has not been addressed.

54 Results: We conducted a double-blind, placebo-controlled, parallel arm study exploring the effects

55 of saccharin on gut microbiota and glucose tolerance in healthy men and women. Participants 56 were randomized to placebo, saccharin, lactisole (STR inhibitor), or saccharin with lactisole 57 administered in capsules twice daily to achieve the maximum acceptable daily intake for two 58 weeks. In parallel, we performed a ten-week study administering high-dose saccharin in the 59 drinking water of chow-fed mice with genetic ablation of STRs (T1R2-KO) and wild-type (WT) 60 littermate controls. In humans and mice alike, none of the interventions affected glucose or 61 hormonal responses to a glucose tolerance test, nor ex vivo glucose absorption in mice. Similarly, 62 saccharin supplementation did not alter microbial diversity or abundance at any taxonomic level 63 in humans or mice. No treatment effects were also noted in readouts of microbial activity such as 64 fecal metabolites or short chain fatty acids (SCFA). However, compared to WT, T1R2-KO mice 65 were protected from age-dependent increases in fecal SCFA and the development of glucose 66 intolerance.

67 Conclusions: In the absence of other permissive conditions, short-term saccharin consumption at 68 the maximum recommended levels does not alter gut microbiota or induce glucose intolerance 69 and, thus, it may be safely included in the diet of healthy individuals who wish to substitute sugars 70 for weight management or caloric control.

71 Trial registration number NCT03032640, Registered 26 January 2017.

72 https://clinicaltrials.gov/ct2/show/NCT03032640

73 Keywords: artificial sweeteners; saccharin; sweet taste receptors; gut microbiota; glucose 74 intolerance; short-chain fatty acids; fecal metabolomics; T1R2; dysbiosis

\section{Background}


Non-caloric artificial sweeteners (NCAS) are often consumed as a substitute for dietary sugars,

77 limiting the caloric content of food without compromising its palatability. Six NCAS are approved as food additives in the United States (saccharin, aspartame, acesulfame potassium, sucralose, neotame, and advantame) by the Food and Drug Administration (FDA). The use of NCAS has increased dramatically over the past decade [1,2], due to growing awareness of the negative health outcomes associated with sugar overconsumption [3]. Strikingly, NCAS use in children has

82 tripled in a decade [4] with recent estimates suggesting that $25 \%$ of children and $41 \%$ of adults in

83 the United States are daily consumers of NCAS [4]. Paradoxically, some epidemiological and experimental studies suggest that consumption of NCAS is associated with metabolic syndrome, weight gain, obesity, and non-alcoholic fatty liver disease (reviewed in $[5,6]$ ). These findings have raised concerns among consumers and health professionals alike that NCAS may not be physiologically inert, as originally thought, and that their general use may lead to adverse public must be answered before concluding that these concerns apply to the general population and, ultimately, setting health policy to guide optimal NCAS use [7]. Among the pathophysiological mechanisms hypothesized to underlie the adverse effects attributed to NCAS consumption, one of the most plausible is the suggestion that consumption of various NCAS such as saccharin, aspartame or sucralose can directly alter gut microbiota composition to cause glucose intolerance in both mice and humans [8]. Indeed, seven days of saccharin supplementation in humans produced dysbiosis and glucose intolerance, but only in a subset of participants that also had a distinct microbiota composition prior to the intervention [8].

97 Similarly in mice, ten weeks of saccharin supplementation caused microbiota-induced glucose 98 intolerance when accompanied by glucose consumption (in the water) or by high fat diet feeding 99 [8]. As both sucrose consumption and a high-fat diet can independently alter the gut microbiome 100 [9], these confounding factors may have played a permissive role allowing the manifestation of 101 NCAS-induced effects on gut microbiota and glucose homeostasis. Due to the absence of 
interventional studies that specifically test the effects of NCAS consumption as an independent modulator of gut microbiome and glucose tolerance, it is unclear whether conclusions from prior studies can be extrapolated to healthy populations that consume NCAS as part of a standard western diet. Finally, it is perplexing how compounds that are unrelated in chemical structure, such as saccharin, aspartame, and sucralose, can cause homogeneous changes in gut microbiota without a common mechanism that involves the host. NCAS are bona fide ligands for sweet taste receptors (STRs) which, beyond the tongue, are expressed in a variety of tissues including the gastrointestinal tract. Intestinal STRs play a role in regulating metabolic responses to the ingestion of sugars [10], so it is reasonable to speculate that STR-mediated chemosensation in the gut may provide a mechanistic link between NCAS-induced metabolic dysfunction and gut microbiota.

To circumvent the limitations of prior studies and explore the potential role of chemosensory STRs in the gut, we conducted a comprehensive translational investigation using humans and rodents. First, we performed a randomized, double-blind, placebo-controlled interventional study during which the diet of healthy participants was supplemented for two weeks with capsules that contained saccharin at the maximum acceptable daily intake (ADI), lactisole (a human specific inhibitor of human STRs), saccharin with lactisole, or placebo. To address potential adverse effects that may require higher NCAS dose and time of exposure and to shed light on possible mechanistic effects of saccharin on gut microbiota and glucose homeostasis, we performed a corresponding study in chow-fed mice with a genetic ablation of STRs (T1R2-KO) or wild-type controls (WT) aiming to exceed the maximum saccharin ADI for 10 weeks.

\section{$\underline{\text { Results }}$}

\section{Human participants}

A total of fifty-four participants were randomized to four treatment groups. Forty-six subjects completed the study and were included in all analyses. Eight participants were excluded from the analysis due to non-compliance (Supp. Figure.1). The clinical characteristics of all participants 
are summarized in Supp. Table.1. At baseline, no differences in basic anthropometric and

129 metabolic parameters were noted between treatment groups (Table.1). The remaining

130 participants of all groups met the expected dose requirement for the treatment period (Supp.

131 Table.2). No adverse effects of the treatments were reported.

132 Glucose tolerance and ex vivo intestinal function

133 Two weeks of continuous saccharin supplementation at a dose equal to ADI [11] did not alter 134 glucose responses to a $75 \mathrm{~g}$ oral glucose tolerance test (OGTT) among participants (Figure.1A).

135 To test for possible delayed effects of the treatment, we assessed glucose tolerance after a two136 week recovery period during which all groups received placebo. No differences in glucose 137 excursions were observed between the post-treatment and recovery (washout) periods (ANCOVA 138 repeated measures $p=0.99$; Supp. Figure.2). Similar to glucose responses, plasma excursions of insulin, C-peptide, glucagon or glucagon-like peptide 1 (GLP-1) were not different between groups with treatment or after the wash out period (Figure.1B-E and Table.2) (Supp. Figure.2).

141 Next, we addressed the long-term effects of high-dose saccharin supplementation on glucose 142 tolerance in mice and specifically explored the role of NCAS sensing by intestinal STRs. Ad libitum 143 chow-fed WT and T1R2-KO mice were supplemented with saccharin in the drinking water for 10 144 weeks to achieve daily consumption equal to 4 times the human ADI adjusted for mouse body 145 surface area [12]. The actual saccharin consumption slightly exceeded the target consumption 146 for both genotypes (Supp. Figure.3A), but without affecting food intake (Supp. Figure.3B). 147 Saccharin consumption did not cause differences in body weight gain compared to water alone 148 in either genotype (Supp. Figure.3C). As we observed in humans, saccharin treatment had no 149 effect on glucose tolerance in WT or T1R2 mice assessed after two or ten weeks of treatment 150 (Figure.2A-B). However, we did observe age-dependent increases in intra-gastric GTT (IGGTT) 151 responses in WT mice. Notably, these effects were absent in T1R2-KO mice, which also had 152 reduced IGGTT responses compared to WT littermates [13] (Figure.2A). 
Although saccharin treatment was unsuccessful in modifying IGGTT responses, it may have

154 induced localized intestinal changes that may contribute to long-term metabolic susceptibility. To

155 address this possibility we assessed post-treatment ex vivo glucose transport using intact 156 intestines (Ussing chamber) and found no effect of saccharin supplementation in the transport of 157 the non-metabolizable glucose analog 3-O-methyl-glucose (3-OMG) (Figure.2C), but we 158 observed decreased glucose transport in T1R2-KO intestines, consistent with the IGGTT data 159 and our previous studies [13]. In addition, saccharin supplementation did not change the 160 expression of glucose transporters or of STRs (Supp. Figure.3D). Because saccharin treatment 161 was shown to disrupt epithelial cell barrier in Caco-2 cell monolayers [14], we assessed ex vivo 162 FITC-dextran (4kDa) flux in treated intact intestines and found no differences in gut permeability 163 (Figure.2D).

\section{Gut microbiota}

165 Saccharin-induced glucose intolerance was previously shown to be contingent upon direct changes in gut microbiota composition [8], so we performed 16S rRNA sequencing of fecal samples from the human and mouse studies to investigate whether alterations in microbial communities are induced in response to treatments despite the absence of metabolic responses. All human subjects had similar microbiota composition prior to the interventions (Supplemental Figure.4A). Also, no sex-dependent differences in Shannon diversity index at baseline were 171 noted (Supplemental Figure.4B). None of the treatments affected relative microbial abundances

172 at any taxonomic levels (Family taxa are shown; Figure.3A) or the degree of microbial diversity 173 (Figure.3B). Multivariate analysis by non-metric multidimensional scaling (NMDS) also showed 174 no clear clustering by treatment (Figure.3C). Taxonomic distributions were equivalent across 175 treatments when we performed pre-post analysis of variance to account for between subjects 176 differences in microbial communities within a treatment group (Two-way ANOVA; $p>0.05$ for each 177 treatment). In mice, we did not observe a genotype or gender effect on gut microbiota composition 178 or taxonomic diversity at baseline (Supplemental Figure.4C-F). Despite the larger dose and 
179 longer duration of treatment in mice, saccharin did not produce any change in microbial 180 abundances at any taxonomic level (Family taxa are shown; Figure.3D) or diversity (Figure.3E). 181 Similar to humans, no clustering effect was observed for post-treatment groups by multivariate 182 analysis (Figure.3F). Also, no changes were noted in within-subject microbial abundances, as 183 assessed by pre-post analysis of variance (Two-way ANOVA; $p>0.05$ for each treatment at family 184 level).

\section{Fecal metabolomics}

186 Although the interventions did not induce substantial shifts in the gut microbial communities in 187 either humans or mice, we tested whether saccharin might have instead altered the intestine's 188 metabolic profile by performing untargeted metabolomics of fecal samples.

189 Multivariate analysis showed that human participants had similar metabolomics profiles at 190 baseline (Supplemental Figure.4G) and none of the interventions affected the fecal metabolome

191 (Figure.4A). Importantly, we did observe saccharin in feces from participants assigned to the corresponding intervention groups, indicating that the saccharin dose was sufficient to reach the intestinal microbiota (Figure.4B). All mice had similar baseline fecal metabolome, excluding 194 potential genotype effect (Supplemental Figure.4H). Initial analysis showed an effect of 195 saccharin treatment compared to water, with a moderate predictive value in the orthogonal partial 196 least squares discriminant analyses (OPLS-DA; Figure.4C). Subsequent metabolite distribution 197 and identification (S-plot analysis) revealed that the presence of saccharin itself in the feces was 198 the only metabolite responsible for the clustering effect (Figure 4D-E). Hence, removal of 199 saccharin from the model abolished the clustering effects, eliminating any independent treatment 200 effects on the fecal metabolome (Figure 4F). In addition, we specifically assessed fecal glucose 201 content in all human and mouse samples, but found no treatment or genotype differences 202 excluding major defects in glucose absorption. (Supplemental Figure.4I-J). Finally, we 203 independently measured short-chain fatty acids (SCFA) in feces and found no treatment effect in 
204 human participants (Figure.4G). However, we noticed an age-dependent increase in SCFA in

205 WT mice, but these effects were absent in T1R2-KO mice (Figure.4H).

206 Discussion

207 Concerns and confusion about the general safety of NCAS can be attributed, in part, to the 208 amount and quality of the available evidence. A critical knowledge gap has been the lack of 209 interventional studies designed to rigorously investigate whether consumption of NCAS per se is 210 sufficient to cause deterioration of glucose homeostasis in healthy individuals. Using a 211 randomized, placebo-controlled design, we clearly show that daily consumption of saccharin at 212 maximum ADI for 2 weeks is inadequate to alter fecal microbiota composition and metabolites or 213 affect glucose tolerance in healthy participants. Notably, identical results were recapitulated in 214 chow fed mice that consumed saccharin equal to 4-times the human ADI for 10 weeks.

215 Over the past 30 years, a number of cross-sectional and observational studies have reported 216 positive correlations between NCAS consumption and outcomes such as metabolic syndrome 217 and weight gain (reviewed in $[6,15]$ ). These findings have alarmed both consumers and health 218 care professionals, despite the fact that health and other lifestyle-related characteristics of the 219 populations might have influenced these outcomes through reverse causality or residual 220 confounding. For instance, positive associations between NCAS consumption (estimated from 221 soda consumption) and metabolic syndrome were noted in a recent cross-sectional study [16], 222 but after careful adjustment for age, dietary quality and physical activity these associations 223 disappeared. A paucity of well-controlled interventional studies has also contributed to confusion 224 in the field.

225 In this regard, an elegant report by Suez et al (2014) [8] appeared to establish a causative 226 relationship between the consumption of NCAS (i.e. saccharin) and the development of glucose 227 intolerance through direct modification of gut microbiota composition. This report, mainly 228 conducted in mice, revived concerns about the use of NCAS and long-term health implications. 229 However, in this study only 3 out of the 7 human participants developed glucose intolerance in 
response to 3-7 days of NCAS use. In contrast, we exposed 23 healthy lean participants in 2 231 separate cohorts (Saccharin, or Saccharin plus Lactisole groups) to 15 days of daily saccharin 232 consumption at the maximum ADI levels. None of the treated subjects, who were also not regular 233 NCAS users, developed glucose intolerance or showed altered endocrine responses during an 234 OGTT, but it is reasonable to speculate that the treatment effects of NCAS supplementation may 235 be delayed. However, OGTT responses remained unaltered after 2 additional weeks of placebo 236 treatment following the main intervention. In agreement with our findings in healthy lean 237 participants, 12 weeks of NCAS supplementation using sweetened beverages did not change 238 glucose tolerance in healthy overweight or obese individuals [17]. This suggests that the 239 development of glucose intolerance in response to NCAS use is independent of obesity status per se and may instead require the presence of other, yet unknown, underlying risk factors. For instance, the saccharin responders in Suez et al (2004) [8] had different baseline microbiome compared to non-responders; a factor shown to confound outcomes of dietary interventions [18]. We circumvented these issues since all participants contained similar basal gut microbiota composition. This similarity is partially due to the enforcement of comprehensive inclusion and exclusion criteria including dietary habits that were consistent with the typical macronutrient intake 246 of healthy US adults. Thus, saccharin treatment did not alter gut microbiota composition 247 compared to other interventions, but also did not induce any relative changes in treated 248 participants (i.e. within-subject pre-post analyses). Although gut microbiota abundances were 249 mainly unaltered by the treatments, marginal shifts in some species or changes in microbial 250 metabolism [19] might predispose the host to dysbiosis [20]. This effect is unlikely, as neither 251 saccharin nor any other treatment significantly altered fecal metabolite profiles or induced any 252 relative changes in treated participants. The microbiota-induced pathophysiology is often linked 253 to SCFA changes in microbial production and availability [21], but saccharin did not alter fecal 254 SCFA in humans and mice alike, mirroring the null effect observed in untargeted metabolite 255 profiles. However, the age-dependent increase in SCFA in the WT mice is consistent with the 
age-dependent development of glucose intolerance in the same mice and it is in agreement with

257 findings showing that increased fecal SCFA correlate with age, obesity and metabolic 258 dysregulation [22]. Notably, in T1R2 mice the absence of SCFA increases with aging correlates 259 with the absence of glucose intolerance. These associations require further investigation since 260 fecal concentrations of SCFA can be affected by several factors including transit time [23] and 261 colonic clearance [24].

262 Interestingly, saccharin was detected in the feces of several saccharin- or saccharin plus lactisole263 treated participants. From a clinical perspective, this observation is very significant because about $26490 \%$ of ingested saccharin is absorbed in the small intestine and eliminated in the urine without 265 biotransformation, while the remainder excreted in the feces [25]. Thus, only a small portion of 266 ingested saccharin can reach and potentially be metabolized by the microbes at the large 267 intestine. Similar to Suez et al [8], we administered saccharin equivalent to the ADI [11], 268 suggesting that saccharin bioavailability was not a limiting factor for gut microbes in our 269 population. Nevertheless, even in high saccharin consumers ( $>90^{\text {th }}$ percentile) the average intake 270 is only about $2 \mathrm{mg} / \mathrm{kg} / \mathrm{d}$, a minor fraction of the ADI $(5 \mathrm{mg} / \mathrm{kg} / \mathrm{d})$ [26]. Taken together with our 271 findings, it is reasonable to suggest that typical saccharin use is unlikely to induce adverse 272 alterations in the gut microbiota of the general healthy consumer.

273 On the other hand, the absence of effects following short-term NCAS supplementation in our 274 study cannot exclude the possibility that the deleterious consequences of NCAS consumption 275 might require higher doses and/or longer durations. Because of safety limitations regarding the 276 dose and duration of treatment involving human participants, we supplemented C57BI/6J mice 277 with saccharin for 10 weeks using a target dose that exceeded the human ADI by 4 times adjusted 278 for mouse body surface area to discern possible mechanistic effects that might have not been 279 apparent in the human study. Surprisingly, but in agreement with the human findings, glucose 280 tolerance, gut microbiota composition and fecal metabolite profiles were unaffected by the higher 281 saccharin dose and extended treatment in chow fed mice. As in humans, saccharin appeared in 
282 the feces of almost all treated mice, confirming saccharin's bioavailability for microbial 283 metabolism. In contrast to our findings, mice fed chow diet and supplemented with $10 \%$ solution 284 of commercial saccharin, which contained $95 \%$ glucose by mass, or mice fed high-fat and 285 supplemented with pure saccharin, developed glucose intolerance mediated by unfavorable 286 changes in gut microbiota [8]. Similarly, 12 weeks of saccharin supplementation in chow-fed $287 \mathrm{ICR} / \mathrm{HaJ}$ mice caused marginal glucose intolerance, but responsive mice also showed increased 288 food intake and weight gain [27]. Our saccharin-fed mice consumed similar amount of chow and 289 experienced the same age-related increases in body weight compared to water control 290 littermates. Taken together, these findings suggest that high saccharin consumption may exert 291 negative health outcomes only in the presence of other permissive conditions such as underlying 292 risk factors, caloric overload, or dietary regiments known to independently alter gut microbiota 293 and induce dysbiosis of the host [28]. Finally, because plasma glucose excretion can be 294 modulated by the rate of intestinal glucose absorption, we tested whether saccharin treatment 295 tampered this process and compensated for systemic effects in glucose homeostasis. No 296 differences in the expression of the main glucose transporters, ex vivo intestinal glucose transport, 297 gut permeability or glucose malabsorption were noted in mice supplemented with saccharin, 298 excluding secondary effects on glucose assimilation.

299 In the presence of other permissive dietary factors, saccharin may be able to cause glucose 300 intolerance by directly altering gut microbiota. However, it is still perplexing how other NCAS, such 301 as aspartame or sucralose, can demonstrate identical effects [8] considering that they share no 302 structural similarities to suggest their intersection of common pathways of microbial metabolism. 303 NCAS are bona fide ligands for STRs, so it is reasonable to speculate that if consumption of all 304 NCAS leads to specific metabolic effects, such as glucose intolerance, a common underlying 305 mechanism should exist. Thus, a secondary aim of our studies was to test whether STR partially 306 mediate the effects of NCAS feeding. Participants that consumed lactisole, a human specific 307 inhibitor of STRs, or mice with genetic ablation of STRs had no differences in glucose tolerance 
or gut microbiota in response to saccharin feeding, which suggests that in the absence of a primary effect of NCAS consumption the role of STR signaling is not apparent. Nevertheless, we observed a genotype effect in mice independent of treatment. T1R2-KO mice had reduced IGGTT responses and ex vivo glucose transport compared to WT littermates, confirming our previous

312 findings [13]. Interestingly, although WT mice developed mild age-related glucose intolerance, 313 T1R2-KO mice were resistant to these effects. We previously showed that T1R2-KO mice were 314 also protected against metabolic derangements induced by high-fat diet [29], suggesting that STR 315 signaling may be involved in age- and diet-dependent deterioration of glucose homeostasis.

316 Although we report no adverse effects of short-term NCAS consumption on the glycemic responses in healthy lean participants and mice, our study has some notable limitations. First, we tested saccharin as a representative NCAS but it is unknown whether our results can be extrapolated to all NCAS. Since the six FDA-approved NCAS have different metabolic fates and bioavailability [30], potential health implications relevant to their consumption must be addressed separately. Second, the duration of treatment in humans was limited to two weeks, which may have been inadequate to induce physiological effects in a healthy young population. This does not preclude the possibility that years of chronic high use of saccharin or of other NCAS may eventually lead to slow maladaptive responses or predispose consumers to the development of disease. Third, we focused on a number of outcomes based on previous reports and specific objectives. Thus, saccharin might have altered other physiological parameters that, if measured,

327 may have helped identify other adverse health conditions linked to NCAS consumption.

\section{Conclusions}

329 We clearly show that short-term saccharin supplementation per se is insufficient to alter gut 330 microbiota or induce glucose intolerance in apparently healthy humans and mice on conventional 331 diets. The clinical significance of our findings should not be underestimated since it emphasizes

332 that the recommended saccharin use is safe for healthy consumers that wish to substitute dietary 333 sugars for weight management or caloric control. Our findings also do not contradict previous 
reports showing harmful effects of saccharin. On the contrary, together they highlight that the

335 potential harmful effects of chronic NCAS use are likely contingent upon permissive physiological

336 or lifestyle features in vulnerable populations. Therefore, for individuals who lack these

337 characteristics - such as those studied here - consumption of NCAS is likely innocuous, but for

338 susceptible populations NCAS use may be contraindicated. Consequently, it is imperative that

339 future studies concentrate in isolating and identifying the critical underlying pathophysiology or

340 conditions that may render specific NCAS as harmful.

\section{$341 \quad$ Methods}

\section{Experimental design}

$343 \quad$ Human Studies

344 We conducted a randomized, placebo-controlled, double-blind, interventional study 345 (NCT02835859) at the Advent-Health Translational Research Institute (TRI) in healthy lean male 346 and female participants who were randomly assigned to four intervention groups. Recruitment, 347 enrollment and all study-related visits, including specimen collection and point-of-care laboratory 348 testing, took place at Advent-Health. Subjects were recruited between January 2017 and February 349 2018. The study was approved by the Institutional Review Board at Advent-Health and all 350 participants signed an informed consent.

351 Healthy men and women 18-45 years of age were recruited from volunteer lists and by social 352 media to participate in the study. Only subjects who consumed less than a can of diet beverage 353 or a spoonful of NCASs weekly (or the equivalent from foods) during the past month, whose body 354 mass index $(\mathrm{BMI}) \leq 25.0 \mathrm{~kg} / \mathrm{m}^{2}$, and who were weight stable $( \pm 3 \mathrm{~kg})$ during the 3 months prior to 355 enrollment were included. Subjects with acute or chronic medical conditions that would 356 contraindicate participation in the research testing or that were taking medications that could 357 potentially affect metabolic function were excluded. Specifically, individuals with diabetes, 358 bariatric surgery, inflammatory bowel disease or a history of malabsorption and pregnant or 
nursing women were excluded. A complete list of inclusion and exclusion criteria are available

360 (Supp. methods).

361 Participants were randomized into four treatment groups and were instructed to consume

362 capsules containing: 1) Pulp filler/placebo (1000mg/day 1) Sodium saccharin (400mg/day), 3)

363 Lactisole (670mg/day) or 4) Sodium saccharin (400mg/day) + lactisole (670mg/day) twice daily

364 for two weeks. A sealed envelope with the randomization allocation sequence (SAS procedure

365 PROC PLAN) was given to the pharmacist who prepared and provided the appropriate treatment.

366 The pharmacist was the only un-blinded member of the study. Diet-related instructions were 367 provided to avoid additional consumption of NCASs for the duration of the study. Participants 368 were asked to give blood samples and stool samples during their visits. The investigation agents, 369 saccharin and lactisole, were formulated in capsules for oral delivery (Compounding Pharmacy, 370 Advent-Health) at the maximum acceptable daily intake (ADI) [11].

371 A schematic of the experimental design is shown in Supp. Figure 5A. At visit 1 (pre-intervention), 372 participants arrived at the TRI after a 10-hour overnight fast omitting breakfast and the following 373 procedures were performed: 1) Stool sample collection. 2) Assessment of dietary compliance; 3) 374 Vital signs; 4) Measurements of weight; 5) Insertion of an intravenous (IV) catheter for blood 375 draws; 6) Baseline blood sampling ( $\mathrm{t}=-10,0 \mathrm{~min})$; 7 ) Oral consumption of a $75 \mathrm{~g}$ glucose solution $376(300 \mathrm{~mL})$ to assess glucose tolerance (i.e. OGTT); 8) OGTT blood sampling $(\mathrm{t}=10,20,30,45$, $37760,90,120,180 \mathrm{~min}) ; 9)$ Participants were provided with 2-week supply of treatment capsules 378 and were instructed to consume 2 capsules a day (morning and evening) with water until the night 379 before their next visit. At visit 2 (post-treatment), the same procedures as listed above were 380 repeated. All groups were subjected to additional 2 weeks of pulp filler/placebo capsule treatment 381 (blinded for participants) and at visit 3 (recovery) the same procedures were performed.

382 Blood was collected in $\mathrm{K}_{2}$ EDTA tubes with a cocktail of protease, esterase and DPP-IV inhibitors 383 (BD ${ }^{\mathrm{TM}}$ P800 blood collection system; BD Bioscience, CA). Glucose concentrations were 
measured by a point of care device (NOVA StatStrip Meter); insulin, C-peptide, total GLP1, and glucagon concentrations by immunoassay (Milliplex Map Kit, Millipore, MA).

\section{Mouse studies}

387 All animal experimental procedures were approved by Institutional Animal Care and Use 388 Committee (IACUC) committee of The Ohio State University. Whole body T1R2 deficient mice 389 (T1R2-KO; a gift of Dr. Zuker) were used with WT littermates back-crossed on the C57BII6J strain 390 for at least 10 generations. After weaning, all mice were housed individually in ventilated caging 391 with limited shared environmental exposure and placed on standard polysaccharide chow diet 392 (Teklad \#2016) for 4-5 weeks. Eight week-old mice were randomly assigned to one of the 393 following treatment groups for additional 10-weeks (Supp. Figure.5B): 1) drinking water only 394 (control), 2) drinking water plus saccharin. All groups were on standard chow diet and saccharin 395 concentrations were adjusted based on pilot studies aiming to a) avoid taste aversive effects $396(<0.3 \%$ saccharin in water $)[31]$, b) ensure equal consumption between genotypes since WT mice 397 can taste saccharin but T1R2-KO cannot, and c) to achieve an average daily dose equal to 4 398 times $(250 \mathrm{mg} / \mathrm{kg})$ the human ADI $(62 \mathrm{mg} / \mathrm{kg})$ adjusted for mouse body surface area [12]. An intra399 gastric GTT (IGGTT) was performed at baseline, week 2 and week 10 of the intervention. Fecal 400 pellets we collected at baseline and at week 10 of the intervention for each mouse. The IGGTT 401 was performed in 5-hour fasted mice (h) which received $1 \mathrm{~g} / \mathrm{kg}$ body weight (BW) of glucose. For 402 the saccharin treated groups, saccharin was maintained in the drinking water during the fasting 403 period prior to testing. A baseline IGGTT was performed the day following the initiation of the 404 interventions to account for possible acute effects of saccharin feeding on the test. Blood glucose 405 was sampled from the tail and analyzed with an AlphaTRAK blood glucose monitoring meter 406 (North Chicago, IL). Glucose tolerance curves over time are shown in absolute values. Area under 407 curve (AUC) was calculated using the trapezoid method adjusted for fasted baselines.

408 Ussing Chamber 
Ex vivo glucose transport was measured in intact intestinal sections by monitoring short-circuit

410 current and measuring ${ }^{14} \mathrm{C}$ isotopic flux of $3-\mathrm{O}-$ methyl-glucose $\left(\left[{ }^{14} \mathrm{C}\right]-3-\mathrm{OMG}\right)$, exactly as 411 described previously [13]. To assess gut permeability, 0.2mg/ml of 4kDa FITC-dextran (Sigma)

412 was added to the donor chamber of pre-equilibrated jejunums and FITC flux to the acceptor side 413 was assessed every $15 \mathrm{~min}$ for $1.5 \mathrm{~h}$ in a fluorimeter at $485 \mathrm{~nm}$ excitation and $528 \mathrm{~nm}$ emission.

\section{$414 \quad$ Fecal Microbiota}

415 Genomic DNA was isolated from mouse and human feces using QiaAmp DNA stool kit (QIAGEN), 416 with an additional step of bead beating for 5 min with $0.1 \mathrm{~mm}$ beads to ensure maximum lysis of 417 bacterial cells. Multiplexed libraries were prepared according to the protocol from Illumina using 418 V3-V4 region of 16S rRNA and HiFi HotStart DNA Polymerase (Kapa Biosystems) for amplification. Final amplified products were quantified by ABI Prism library quantitation kit (Kapa Biosystems). Each sample was diluted to $10 \mathrm{nM}$, and equal volume from each sample was pooled.

421 The quality of the library was checked by Bio-Rad Experion bioanalyzer (Bio-Rad). Illumina MiSeq 422 platform was used for sequencing (Novogene Bioinformatics Technology Co., Ltd).

423 Raw FASTQ sequences were quality checked with FastQC v0.11.5. Raw sequences were 424 trimmed with 'cutadapt' v2.6 to remove low quality bases and adaptor sequences. The trimmed 425 FASTQ files were converted into a Qiime2 v2019.1 file format PairedEndFastqManifestPhredd33. 426 The imported forward and reverse reads were merged using 'vsearch' with a minimum sequence 427 length of 200 base pairs. Joined pairs were quality trimmed using Qiime2 'quality filter' with an 428 average quality score of 20 (Q20) over a 3 base pair sliding window and removing trimmed reads 429 having less than $75 \%$ of their original length. 'Deblur 16 S rRNA positive filter' was used as a final 430 quality control step by dereplicating and removing chimera sequences from each sample; reads 431 were trimmed to a final length of 195 base pairs. Taxonomic analysis and Operational Taxonomic 432 Unit (OTU) tables were created with Qiime2 and converted using biom format is Qiime1. All 433 statistics were ran in Graphpad Prism v8 unless specified otherwise. Alpha and beta diversity 434 measurements were calculated using Microbiomeanalyst.ca with no filtering. Alpha diversity 
calculations were based on Shannon diversity index with Mann-Whitney test and figures were plotted in Graphpad Prism. All boxplot data were evaluated with median and minimum/maximum values. Statistical analysis of the multiple group comparisons was performed using one-way analysis of variance (ANOVA) followed by Tukey post-hoc test; when two groups were compared, the nonparametric t-test was performed. For mouse genotypes at 0 weeks, a one-way ANOVA with Tukey post-hoc test was performed to determine initial genotypic effects on microbiome. Results were considered significant with P-value $<0.05$. Beta diversity was calculated on $16 S$ rRNA OTU data using Bray-Curtis dissimilarity and NMDS figure created using R package 'vegan'. Permutational multivariate ANOVA based on NMDS ordination distances was used to calculate community composition. Based on OTU data produced by Qiime2, a relative abundance bar chart was created using Microbiomeanalyst.ca. For abundances statistical analysis, each individual in human and mouse population was tested with a t-test and two-way ANOVA for each family level classification for pre and post treatment.

\section{Fecal Metabolomics}

The nuclear magnetic resonance (NMR) spectra of aqueous fecal extracts were acquired at 298K on a Bruker Avance III $800 \mathrm{MHz}$ spectrometer equipped with a TCl probe (Bruker Biospin, Germany). The ${ }^{1} \mathrm{D}{ }^{1} \mathrm{H}$ NMR experiments were conducted using the first increment of the nuclear Overhauser enhancement spectroscopy (NOESY) pulse sequence with presaturation for water suppression (Relaxation delay-90-t1-90-mixing time-90-Free induction decay). The acquisition parameters were as follows: 64 scans and 4 dummy scans, $64 \mathrm{~K}$ data points, $90^{\circ}$ pulse angle (11.3 us), relaxation delay of $3 \mathrm{~s}$ and a spectral width of $14 \mathrm{ppm}$. The spectra were acquired without spinning the NMR tube in order to avoid spinning side bands artifacts. The free induction decays were multiplied by a decaying exponential function with a $1 \mathrm{~Hz}$ line broadening factor prior to Fourier transformation. The ${ }^{1} \mathrm{H}$ NMR spectra were corrected for phase and a polynomial fourthorder function was applied for base-line correction. Chemical shifts are reported in ppm as referenced to Trimethylsilylpropanoic acid $(\delta=0)$. NMR signal were assigned using a range of 
2D NMR spectra, namely ${ }^{1} \mathrm{H}-{ }^{1} \mathrm{H}$ correlation spectroscopy, ${ }^{1} \mathrm{H}-{ }^{1} \mathrm{H}$ total correlation spectroscopy ,

$462{ }^{1} \mathrm{H}-{ }^{13} \mathrm{C}$ edited heteronuclear single quantum correlation, and ${ }^{1} \mathrm{H}-{ }^{13} \mathrm{C}$ heteronuclear multiple bond 463 correlation spectra. 1D and 2D NMR spectra were processed using TopSpin 3.2 (Bruker Biospin, 464 Germany).

465 The spectral region $\delta 0.50-10.0$ was integrated into regions with equal width of 0.005 ppm using 466 the AMIX software package (V3.8, Bruker-Biospin). The region $\delta 4.70-4.90$ was discarded due 467 to imperfect water saturation. Prior to statistical data analysis, each bucketed region was 468 normalized to the total sum of the spectral intensities to compensate for the overall concentration 469 differences.

470 Multivariate statistical analysis was carried out with SIMCA-P+ software (version 14.1, Umetrics, 471 Sweden). Data were mean-centered and scaled using the Pareto method, while log472 transformation was applied to achieve an improved normal distribution of the data. Principal 473 component analysis (PCA) and orthogonal projection to latent structures with discriminant 474 analysis (OPLS-DA) were conducted on the scaled data. The OPLS-DA model's confidence level 475 for membership probability was set to $95 \%$ and was validated using a 7 -fold cross validation 476 method. The quality of the model was assessed by the values of $R^{2} Y$ and $Q^{2}$. The $R^{2} Y$ metric 477 describes the percentage of variation explained by the model; $Q^{2}$ shows the predictive ability of 478 the model. The difference between these metrics describes the model's fitness.

\section{Fecal Short Chain Fatty Acids}

480 Liquid chromatography tandem mass spectrometry (LC-MS/MS) methods for SCFA were 481 performed as described [32]. Briefly, samples of mouse and human feces were thawed on ice. 482 Samples were then homogenized in $50 \%$ acetonitrile, containing ${ }^{13} \mathrm{C}$-propionate as an internal 483 standard at a ratio of $10 \mu \mathrm{L}$ solvent per $1 \mathrm{mg}$ fecal sample. Fecal samples were then derivatized 484 as described previously [32]. Samples were sealed and stored at $4{ }^{\circ} \mathrm{C}$ until analyses, and 485 throughout LC-MS/MS quantification. All LC-MS/MS analyses were performed within 24 hours of 486 sample creation. Samples were analyzed on an Agilent 6460 QQQ LC-MS/MS system, using a 
Poroshell EC-C18 column $(3.0 \times 50 \mathrm{~mm})$. Collision energies were 10 for butyric acid, 5 for

488 propionic acid, and 15 for acetic acid. Retention times and mass transitions for each SCFA 489 monitored were: Butyrate: 7.138 min., $222 \rightarrow 137$; Propionate: 5.097 min., $208 \rightarrow 165$, 208 $\rightarrow 137$;

$490 \quad{ }^{13}$ C Propionate: 5.097 min., $209 \rightarrow 165,209 \rightarrow 137$; Acetate: 2.754 min., $194 \rightarrow 137$. SCFA levels

491 were quantified using standard curves generated using authentic standards and normalized using

$492{ }^{13} \mathrm{C}$ propionate as an internal standard. Data was analyzed using the Agilent MassHunter

493 Quantitative Analysis software suite.

494 Gene expression

495 Gene expression of scraped mucosa from mouse intestines was performed as described [13]

496 using the following genes: t1r2 (forward: GAACTGCCCACCAACTACAA, reverse:

497 CCATCGTGGACAGACATGAA), t1r3 (forward: CCAGTGAGTCTTGGCTGACA, reverse:

498 TTCAGTGAGGCACAGAATGC), sglt1 (forward: TGGAGTCTACGCAACAGCAAGGAA, reverse:

499 AGCCCACAGAACAGGTCATATGCT), glut2 (forward: CCCTGGGTACTCTTCACCAA, reverse:

500 GCCAAGTAGGATGTGCCAAT).

501 Statistical analyses

502 For human studies, sample size calculation (PROC GLMPOWER, SAS) was based on the

503 minimal detectable difference of glycemic responses (area under curve) during an OGTT 504 performed before and after 7-days of saccharin treatment (Figure.4B of reference [8]), using an 505 ANCOVA model with baseline as covariate to provide $80 \%$ statistical power for one-sided 0.05 506 significance level test. Differences between groups in glycemic and hormonal responses (i.e. 507 AUC) during the OGTT were tested via ANCOVA with the baseline AUC as the covariate, followed 508 by post-hoc multiple comparisons. To investigate the treatment effect at the different visits, we 509 built repeated measures ANCOVA with treatment, time and treatment $x$ time interaction as main 510 effects, along with baseline AUC as a covariate, followed by post-hoc multiple comparisons. For 511 mouse studies, differences between groups in glycemic responses during the OGTT and ex vivo 512 intestinal transport and gene expression were tested by two-way ANOVA. A p-value $<0.05$ was 
513 considered statistically significant. All analyses will be performed with SAS version 9.4 (SAS

514 Institute Inc).

515 Abbreviations

516 3-OMG 3-O-methyl-glucose

517 ADI Acceptable daily intake

518 ANCOVA Analysis of covariance

519 ANOVA Analysis of variance

520 AUC Area under the curve

521 BW Body weight

522 FDA Food and Drug Administration

523 GLP-1 Glucagon-like peptide 1

524 IGGT Intragastric glucose tolerance test

$525 \quad$ KO knockout

526 NCAS Non-caloric artificial sweeteners

527 NMDS non-metric multidimensional scaling

528 OGTT Oral glucose tolerance test

529 OPLS-DA Ortigonal partial least squares discriminant analysis

530 OTU Operational taxonomic unit

531 SCFA Short term fatty acids

532 STRs Sweet taste receptors

533 TRI Translational research institute (Advent-Health)

534 WT Wild-type

535 Declarations:

536 Ethics approval and consent to participate

537 The clinical study was performed in accordance with the requirements of Good Clinical Practice

538 and the Revised Declaration of Helsinki. All participants provided written informed consent to 
participate after receiving verbal and written information about the study. The protocol was

540 approved by the Institutional Review Board of Advent-Health and registered at IRBNet (\#982524).

541 The study was registered on ClinicalTrials.gov on the $26^{\text {th }}$ of January of 2017 (NCT03032640).

542 All the studies in mice were performed in accordance to NIH and institutional guidelines of the

543 Ohio State University Institutional Animal Care and Use Committee.

544 Consent for publication

545 Not applicable

546 Availability of data and materials

547 The raw sequence data from 16S rRNA gene amplicon sequencing were submitted to NCBI

548 BioProject under accession number PRJNA605207

549 https://www.ncbi.nlm.nih.gov/bioproject/PRJNA605207

550 Competing interests

551 The authors declare no competing interests.

$552 \quad$ Funding

553 This work was supported by the National Institutes of Health (R21DK110489 to GAK), the National

554 Institute of Food and Agriculture (NIFA-2018-67001-28246 to GAK), and Advent-Health

555 institutional funds to GAK.

556 Author contributions

557 JS designed research studies, performed experiments, analyzed data, and wrote the manuscript.

$558 \mathrm{KRS}, \mathrm{VS}, \mathrm{JB}, \mathrm{EH}$, performed experiments and analyzed data. VV, TEL, LMD, VS, FT, LG

559 performed experiments. ALC, FY, SNP, MA analyzed data and edited the manuscript. REP

560 designed research studies and edited the manuscript. GAK conceived the project, designed

561 research studies, analyzed data, and wrote the manuscript.

562 Acknowledgments

563 We thank Dr. Daniel J Spakowicz and Rebecca Hoyd (OSU) for technical assistance with data 564 analysis; Dr. Charles Zuker (Columbia University) for mutant mice; Gary Souders (Advent-Health) 
565 for treatment preparation and allocation, Susann Nagel Buller (Advent-Health) for clinical study

566 coordination, and Joshua Smith (Advent-Health) for human sample collection and processing.

\section{References}

568 1. National Center for Health Statistics. Prevalence of overweight, obesity and extreme 569 obesity among adults: United States, trends 1960-62 through 2005-2006. National Health and Nutrition Examination Survey. 2006.

$5712 . \quad$ Sylvetsky AC, Welsh JA, Brown RJ, and Vos MB. Low-calorie sweetener consumption is $572 \quad$ increasing in the United States. The American journal of clinical nutrition. 2012;96(3):6405736.

574 3. Lustig RH, Schmidt LA, and Brindis CD. Public health: The toxic truth about sugar. Nature. 575 576 2012;482(7383):27-9.

4. Sylvetsky AC, Jin Y, Clark EJ, Welsh JA, Rother KI, and Talegawkar SA. Consumption of Low-Calorie Sweeteners among Children and Adults in the United States. J Acad Nutr Diet. 2017;117(3):441-8.e2.

579

5. Green CHS, Wing-Kin. Non-nutritive sweeteners and their association with the metabolic syndrome and non-alcoholic fatty liver disease: a review of the literature | SpringerLink. 2019.

6. Swithers SE. Artificial sweeteners produce the counterintuitive effect of inducing metabolic derangements. Trends in endocrinology and metabolism: TEM. 2013;24(9):431-41.

584 7. Borges MC, Louzada ML, de Sa TH, Laverty AA, Parra DC, Garzillo JM, et al. Artificially Sweetened Beverages and the Response to the Global Obesity Crisis. PLoS Med.

587 8. Suez J, Korem T, Zeevi D, Zilberman-Schapira G, Thaiss CA, Maza O, et al. Artificial 588 589 sweeteners induce glucose intolerance by altering the gut microbiota. Nature. 2014;514(7521):181-6. 
590 9. Parks BW, Nam E, Org E, Kostem E, Norheim F, Hui ST, et al. Genetic control of obesity and gut microbiota composition in response to high-fat, high-sucrose diet in mice. Cell

10. Calvo SS, and Egan JM. The endocrinology of taste receptors. Nat Rev Endocrinol. 2015;11(4):213-27.

595 11. Evaluation of certain food additives and contaminants. Forty-first report of the Joint 596

12. Nair AB, and Jacob S. A simple practice guide for dose conversion between animals and 599

15. Azad MB, Abou-Setta AM, Chauhan BF, Rabbani R, Lys J, Copstein L, et al. Nonnutritive sweeteners and cardiometabolic health: a systematic review and meta-analysis of randomized controlled trials and prospective cohort studies. CMAJ. 2017;189(28):E929E39.

16. Hess EL, Myers EA, Swithers SE, and Hedrick VE. Associations Between Nonnutritive Sweetener Intake and Metabolic Syndrome in Adults. J Am Coll Nutr. 2018;37(6):487-93.

17. Higgins KA, and Mattes RD. A randomized controlled trial contrasting the effects of 4 lowcalorie sweeteners and sucrose on body weight in adults with overweight or obesity. The American journal of clinical nutrition. 2019;109(5):1288-301. 
615 18. Healey GR, Murphy R, Brough L, Butts CA, and Coad J. Interindividual variability in gut microbiota and host response to dietary interventions. Nutr Rev. 2017;75(12):1059-80.

617 19. Zierer J, Jackson MA, Kastenmuller G, Mangino M, Long T, Telenti A, et al. The fecal

20. Eng A, and Borenstein E. Taxa-function robustness in microbial communities. Microbiome. 2018;6(1):45.

621 21. Rios-Covian D, Ruas-Madiedo P, Margolles A, Gueimonde M, de Los Reyes-Gavilan CG, and Salazar N. Intestinal Short Chain Fatty Acids and their Link with Diet and Human Health. Frontiers in microbiology. 2016;7:185.

22. de la Cuesta-Zuluaga J, Mueller NT, Alvarez-Quintero R, Velasquez-Mejia EP, Sierra JA, 625 Corrales-Agudelo V, et al. Higher Fecal Short-Chain Fatty Acid Levels Are Associated with Gut Microbiome Dysbiosis, Obesity, Hypertension and Cardiometabolic Disease Risk Factors. Nutrients. 2018;11(1).

23. Vogt JA, and Wolever TM. Fecal acetate is inversely related to acetate absorption from 629 the human rectum and distal colon. The Journal of nutrition. 2003;133(10):3145-8.

24. Stumpff F. A look at the smelly side of physiology: transport of short chain fatty acids. Pflugers Arch. 2018;470(4):571-98.

632 25. Renwick AG. The disposition of saccharin in animals and man--a review. Food and chemical toxicology : an international journal published for the British Industrial Biological Research Association. 1985;23(4-5):429-35.

26. Renwick AG. The intake of intense sweeteners - an update review. Food Addit Contam.

637 27. Shi Q, Cai L, Jia H, Zhu X, Chen L, and Deng S. Low intake of digestible carbohydrates 638 ameliorates duodenal absorption of carbohydrates in mice with glucose metabolism disorders induced by artificial sweeteners. J Sci Food Agric. 2019;99(11):4952-62. 
640

641

642

643

644

645

646

647

648

649

650

651

652

653

654

655

656

657

658

659

660

661

662

663

664

665

28. Gentile CL, and Weir TL. The gut microbiota at the intersection of diet and human health. Science. 2018;362(6416):776-80.

29. Smith KR, Hussain T, Karimian Azari E, Steiner JL, Ayala JE, Pratley RE, et al. Disruption of the sugar-sensing receptor T1R2 attenuates metabolic derangements associated with diet-induced obesity. American journal of physiology Endocrinology and metabolism. 2016;310(8):E688-E98.

30. Magnuson BA, Carakostas MC, Moore NH, Poulos SP, and Renwick AG. Biological fate of low-calorie sweeteners. Nutr Rev. 2016;74(11):670-89.

31. Sclafani A, Bahrani M, Zukerman S, and Ackroff K. Stevia and saccharin preferences in rats and mice. Chemical senses. 2010;35(5):433-43.

32. Han J, Lin K, Sequeira $\mathrm{C}$, and Borchers $\mathrm{CH}$. An isotope-labeled chemical derivatization method for the quantitation of short-chain fatty acids in human feces by liquid chromatography-tandem mass spectrometry. Anal Chim Acta. 2015;854:86-94.

\section{Figure Legends}

Figure 1. Effects of saccharin and/or lactisole treatment on glucose tolerance in humans Plasma excursions of (A) glucose, (B) insulin, (C) C-peptide, (D) glucagon, and (E) GLP-1 during an oral glucose challenge after 2 weeks of treatment. Two-way ANOVA repeated measures $(p>0.05) . N=10-13$.

Figure 2. Effects of saccharin treatment on glucose homeostasis in mice (A) Glucose responses during an i.g.GTT expressed as area under curve (AUC) before (0 weeks), 2 and 10 weeks after saccharin treatment. Two-way ANOVA main effect; * $p<0.05$, WT 10-week vs. T1R2 10-week, ${ }^{* *}$ p<0.01 WT 0-week vs. WT 10-week). (B) Glucose excursions during an i.g.GTT in response to 10 weeks of saccharin treatment (2-way ANOVA repeated measures, $\mathrm{p}=0.0007)$. (C) Ex vivo glucose flux using 3-O-methy-glucose (3-OMG) in intact mouse intestines following 10 weeks of saccharin treatment. Two-way ANOVA, ${ }^{* *} p<0.01$. (D) Ex vivo intestinal permeability assessed by FITC-dextran $(4 \mathrm{kDa})$ flux in intact mouse intestines 
666

667

668

669

670

671

672

673

674

675

676

677

678

679

680

681

682

683

684

685

686

687

688

689

690

691

following 10 weeks of saccharin treatment. Two-way ANOVA, $p>0.05 . \mathrm{N}=23-28$ for in vivo studies, $\mathrm{n}=6-11$ for ex vivo studies.

Figure 3. Taxonomic abundances and diversity of gut microbiota in response to treatments in humans and mice

(A and D) Bar chart summary showing relative abundance at the family level post-treatment in human participants or in WT and T1R2 mice. Each bar represents abundances of one subject.

(B and E) Alpha diversity box plot (Shannon diversity metric) showing community richness between groups post-treatment in human participants (Mann-Whitney $U$ Test; $p=0.156, U=5.22$ ) or in WT and T1R2 mice (Mann-Whitney $U$ Test; $p=0.987, U=152$ ). (C and F) Nonmetric multidimensional scaling (NMDS) plot showing community similarities between groups posttreatment in human participants $(p<0.999$, NMDS stress $=0.2274)$ or WT and T1R2 mice $(p<$ 0.111 , NMDS stress $=0.209$ ). NMDS ordination was derived from pairwise Bray-Curtis distances and statistical inferences made using PERMANOVA. $\mathrm{N}=11-13$ for human studies, $\mathrm{n}=8-11$ for mouse studies.

Figure 4. Fecal metabolomics in response to treatments in humans and mice

(A) Differences in human fecal metabolites between treatment groups using orthogonal partial least squares discriminant analyses (OPLS-DA). (B) Post-treatment saccharin presence in human fecal samples. Dashed lines represent average noise \pm SD. (C) Differences in WT and T1R2 fecal metabolites following saccharin treatment using OPLS-DA. (D) Post-treatment saccharin presence in mouse fecal samples. Dashed lines represent average noise \pm SD. Metabolite distribution (S-plot) in fecal mouse samples. Metabolites attributed to saccharin shown in red. (F) Differences in WT and T1R2 fecal metabolites following saccharin treatment using OPLS-DA after removal of saccharin signals. (G) Assessment of short chain fatty acids (SCFA) following treatments in human samples. One-way ANCOVA baseline as covariate. (H) SCFA in mouse feces before (pre) and after (post) treatment. Two-way ANOVA repeated measures with post-hoc. $\mathrm{N}=11-13$ for human studies, $\mathrm{n}=8$ for mouse studies. 
TABLE 1

Baseline characteristics of intervention groups

\begin{tabular}{|c|c|c|c|c|c|}
\hline & Placebo & Saccharin & Lactisole & $\mathrm{Sac}+\mathrm{Lac}$ & \\
\hline Total, $\mathrm{n}$ & & 1 & 13 & 12 & $\mathrm{p}$ \\
\hline Age, y & $24.91 \pm 1.59$ & $28.91 \pm 2.60$ & $32.92 \pm 2.78$ & $28.80 \pm 2.91$ & 0.199 \\
\hline Height, cm & $166.61 \pm 2.37$ & $169.03 \pm 3.31$ & $164.54 \pm 1.92$ & $172.53 \pm 2.23$ & 0.494 \\
\hline Weight, kg & $59.00 \pm 1.81$ & $64.52 \pm 3.49$ & $62.13 \pm 1.90$ & $66.57 \pm 2.64$ & 0.305 \\
\hline BMI, kg/m2 & $21.29 \pm 0.62$ & $22.40 \pm 0.53$ & $22.93 \pm 0.47$ & $22.38 \pm 0.78$ & 0.261 \\
\hline Glucose, mg/dL & $87.55 \pm 2.40$ & $92.00 \pm 2.41$ & $91.63 \pm 2.64$ & $90.00 \pm 1.23$ & 0.519 \\
\hline Triglycerides, mg/dL & $72.36 \pm 7.93$ & $71.82 \pm 13.88$ & $87.42 \pm 12.85$ & $65.70 \pm 7.57$ & 0.605 \\
\hline Total cholesterol, mg/dL & $166.91 \pm 9.38$ & $163.82 \pm 10.09$ & $182.33 \pm 8.25$ & $154.60 \pm 7.47$ & 0.333 \\
\hline $\mathrm{HDL}, \mathrm{mg} / \mathrm{dL}$ & $66.55 \pm 4.12$ & $57.55 \pm 3.74$ & $62.58 \pm 4.60$ & $64.80 \pm 3.96$ & 0.236 \\
\hline LDL, mg/dL & $85.91 \pm 7.92$ & $91.82 \pm 7.47$ & $102.25 \pm 7.29$ & $76.50 \pm 9.24$ & 0.162 \\
\hline Cholesterol/HDL & $2.58 \pm 0.18$ & $2.95 \pm 0.27$ & $3.08 \pm 0.28$ & $2.49 \pm 0.20$ & 0.103 \\
\hline LDL/HDL & $1.36 \pm 0.17$ & $1.67 \pm 0.22$ & $1.77 \pm 0.25$ & $1.26 \pm 0.19$ & 0.115 \\
\hline
\end{tabular}

All values are mean \pm SEM. Baseline differences between groups were assessed by ANCOVA using sex as covariate. BMI, body mass index; HDL, high density cholesterol; LDL, low density cholesterol. AUC, area under the curve; Sac, saccharin; Lac, lactisole. 
Figure 1
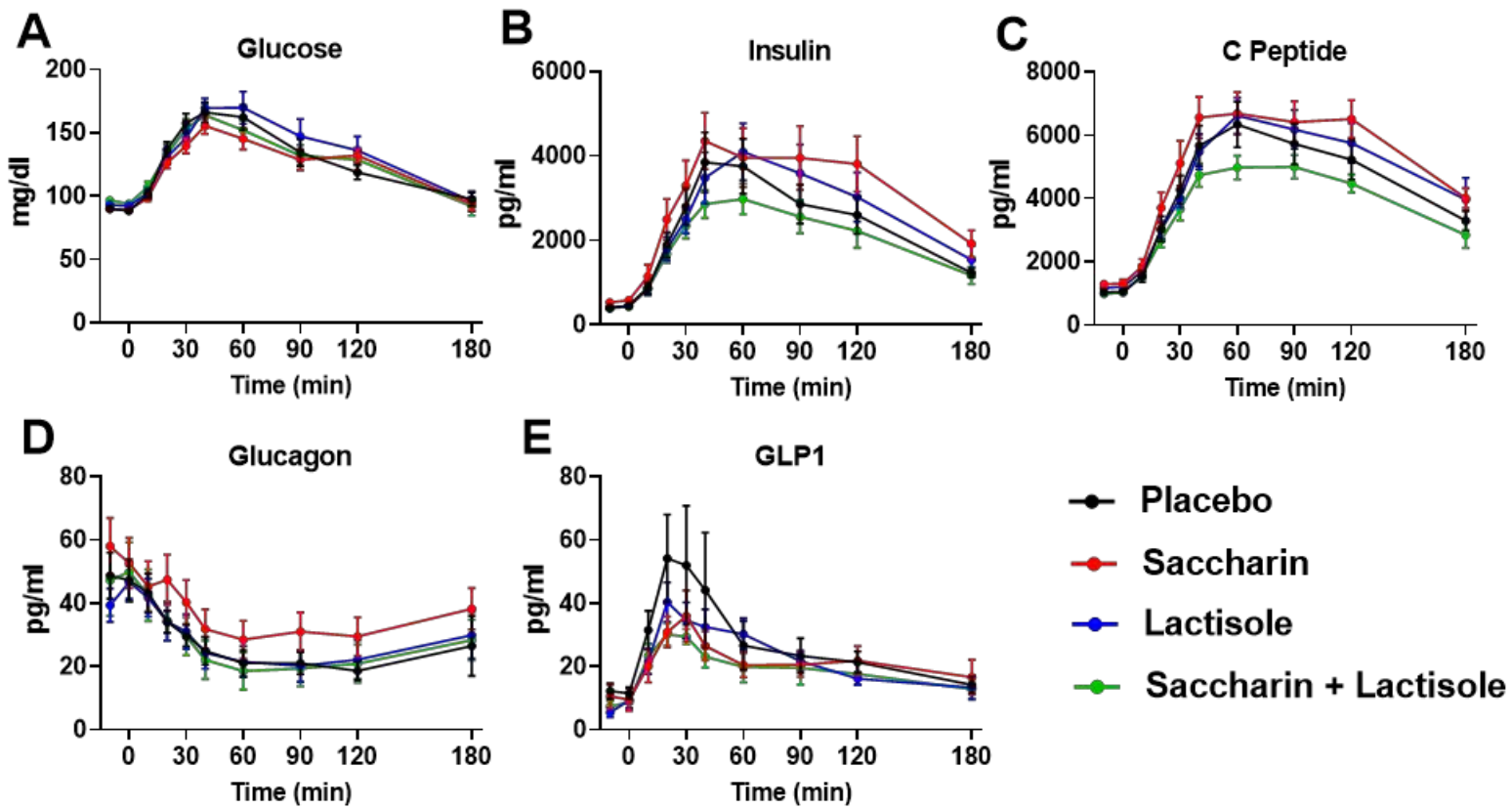


\section{Table 2.}

\section{TABLE 2}

Glucose and hormonal excursions during an OGTT after the intervention

\begin{tabular}{llllll}
\hline & Placebo & Saccharin & Lactisole & Sac + Lac & $\mathrm{p}$ \\
\hline Glucose (AUC) & $7136.4 \pm 943.6$ & $6606.9 \pm 951.7$ & $7571.0 \pm 1289.2$ & $6203.8 \pm 1084.2$ & 0.6018 \\
Insulin (AUC) & $362518.7 \pm 55694.9$ & $476124.6 \pm 77735.0$ & $412000.5 \pm 69663.6$ & $299078.8 \pm 41026.4$ & 0.7627 \\
C peptide (AUC) & $645605.0 \pm 73479.8$ & $747311.4 \pm 68855.8$ & $677199.5 \pm 61852.4$ & $525798.3 \pm 28535.9$ & 0.6034 \\
Glucagon (AUC) & $-3993.5 \pm 771.9$ & $-3226.6 \pm 1306.1$ & $-3768.0 \pm 984.4$ & $-4504.1 \pm 1528.9$ & 0.8632 \\
GLP1 (AUC) & $2734.8 \pm 983.2$ & $2195.4 \pm 410.7$ & $2294.1 \pm 384.5$ & $1862.9 \pm 520.9$ & 0.0662 \\
\hline
\end{tabular}

All values are mean \pm SEM. Treatment effects between groups were assessed by ANCOVA using the baseline glucose tolerance test AUC as a covariate. OGGT, oral glucose tolerance test; AUC, area under curve; Sac, saccharin; Lac, lactisole 
Figure 2

A

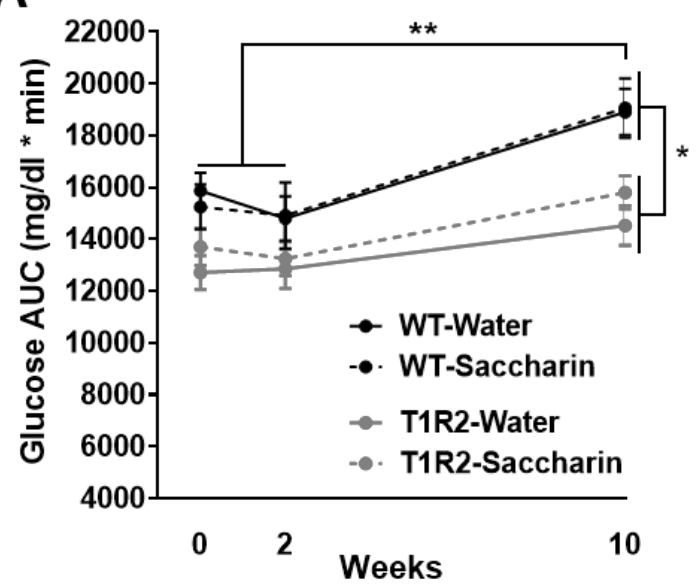

B

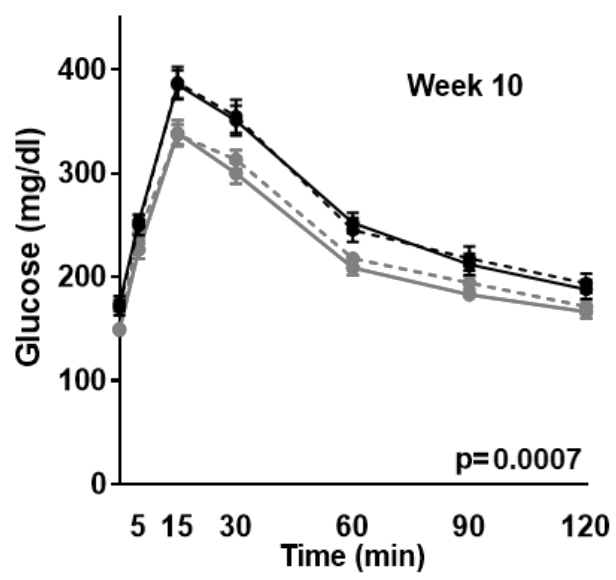

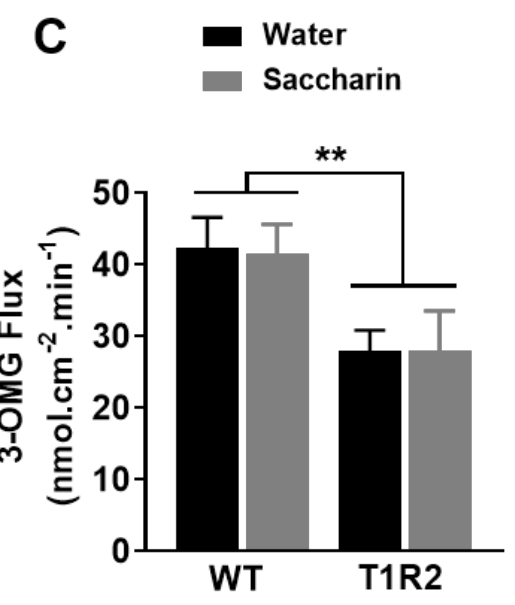

D

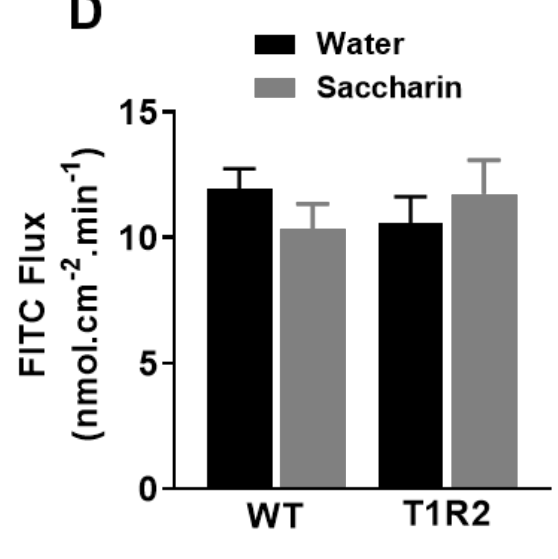




\section{Figure 3}

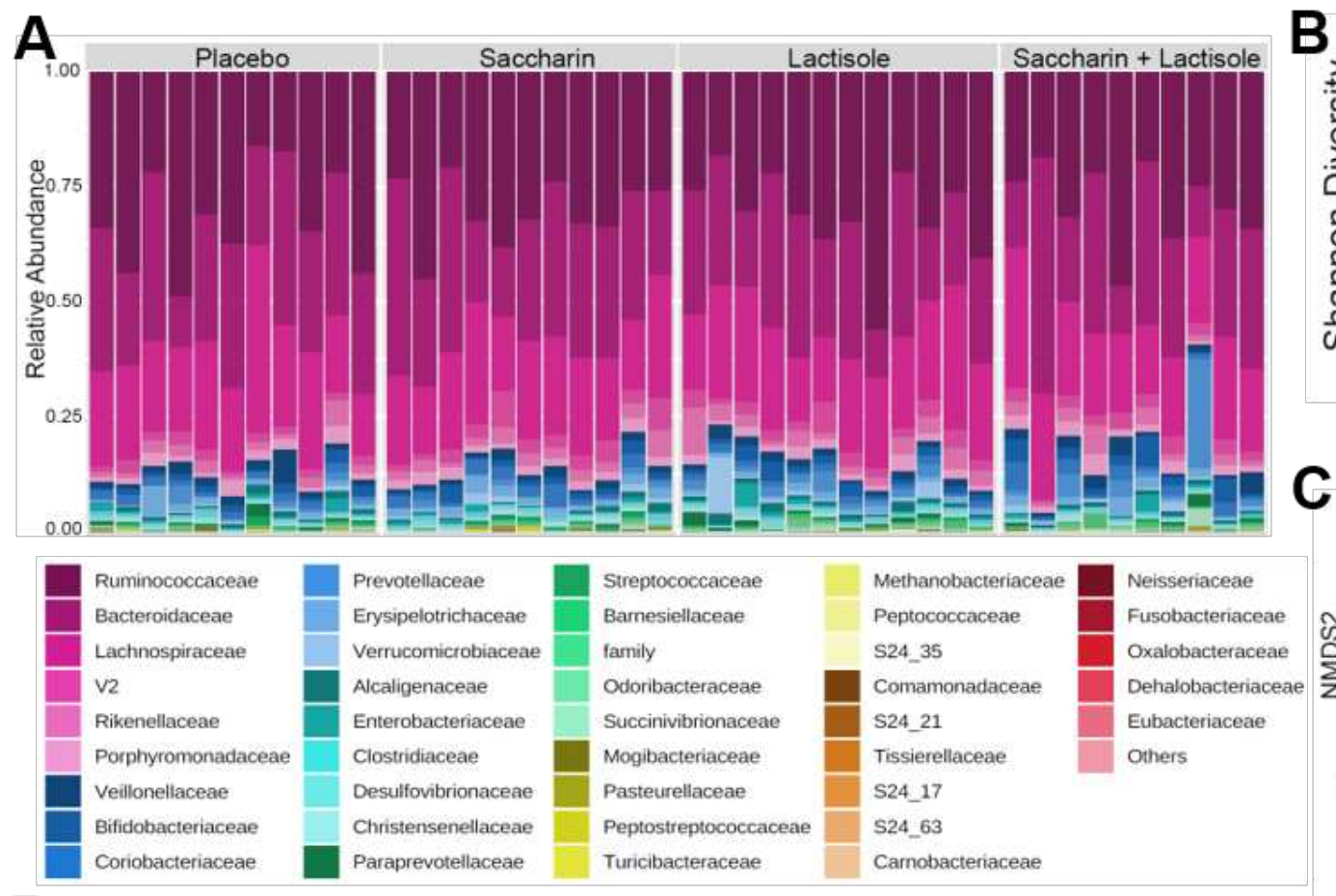

B

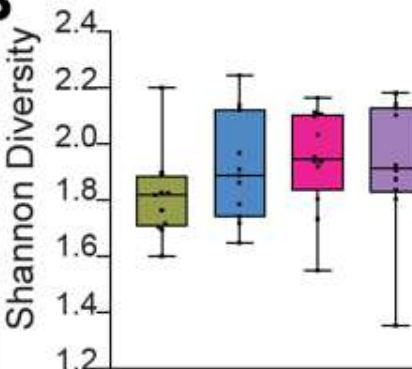

\section{D}
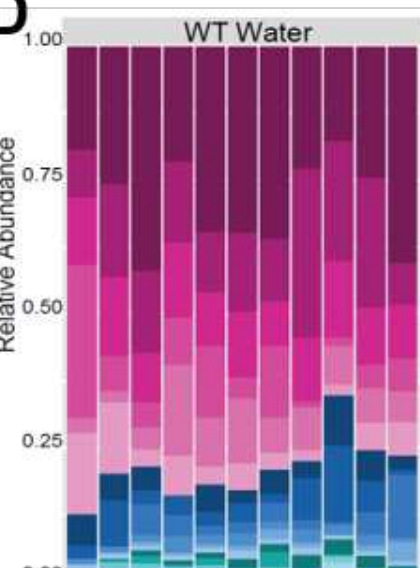
$0.00=-2=$
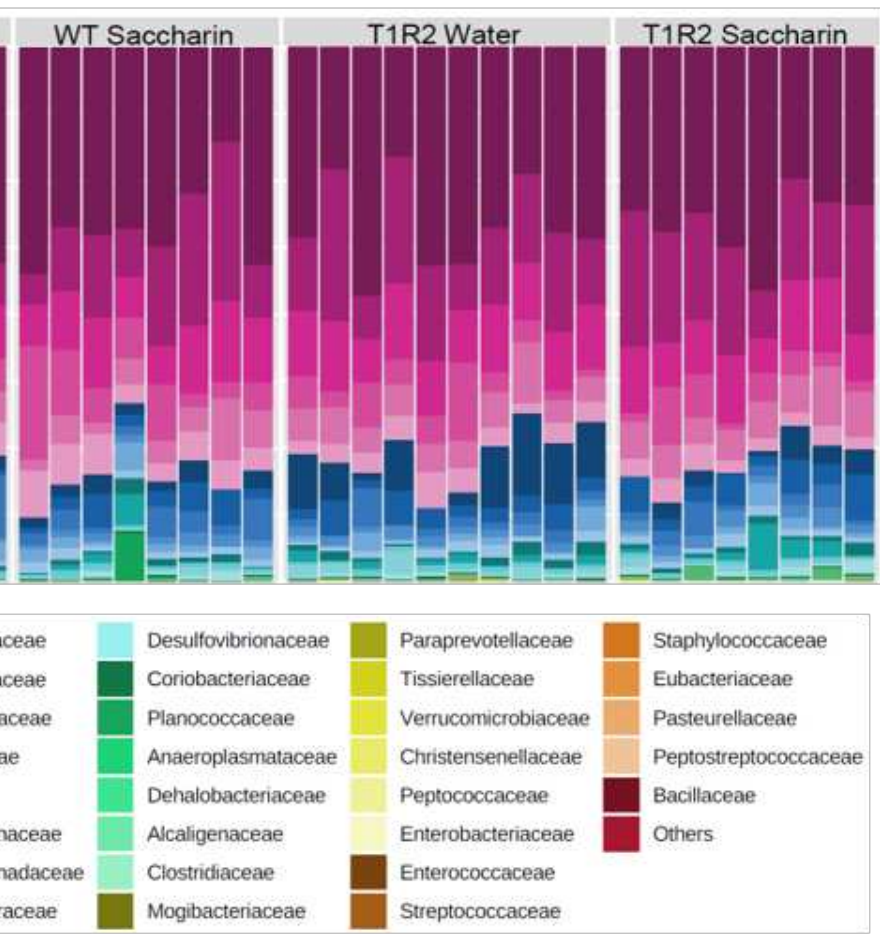

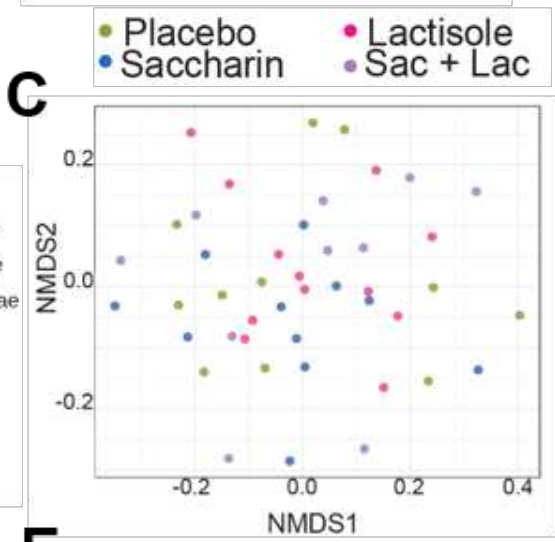

E

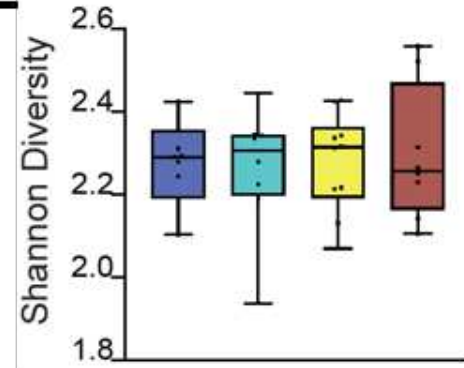

-WT Water T1R2 Water

-WT Saccharin -T1R2 Saccharin

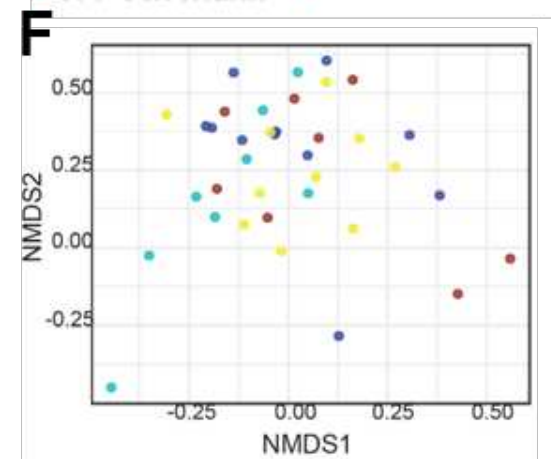


Figure 4
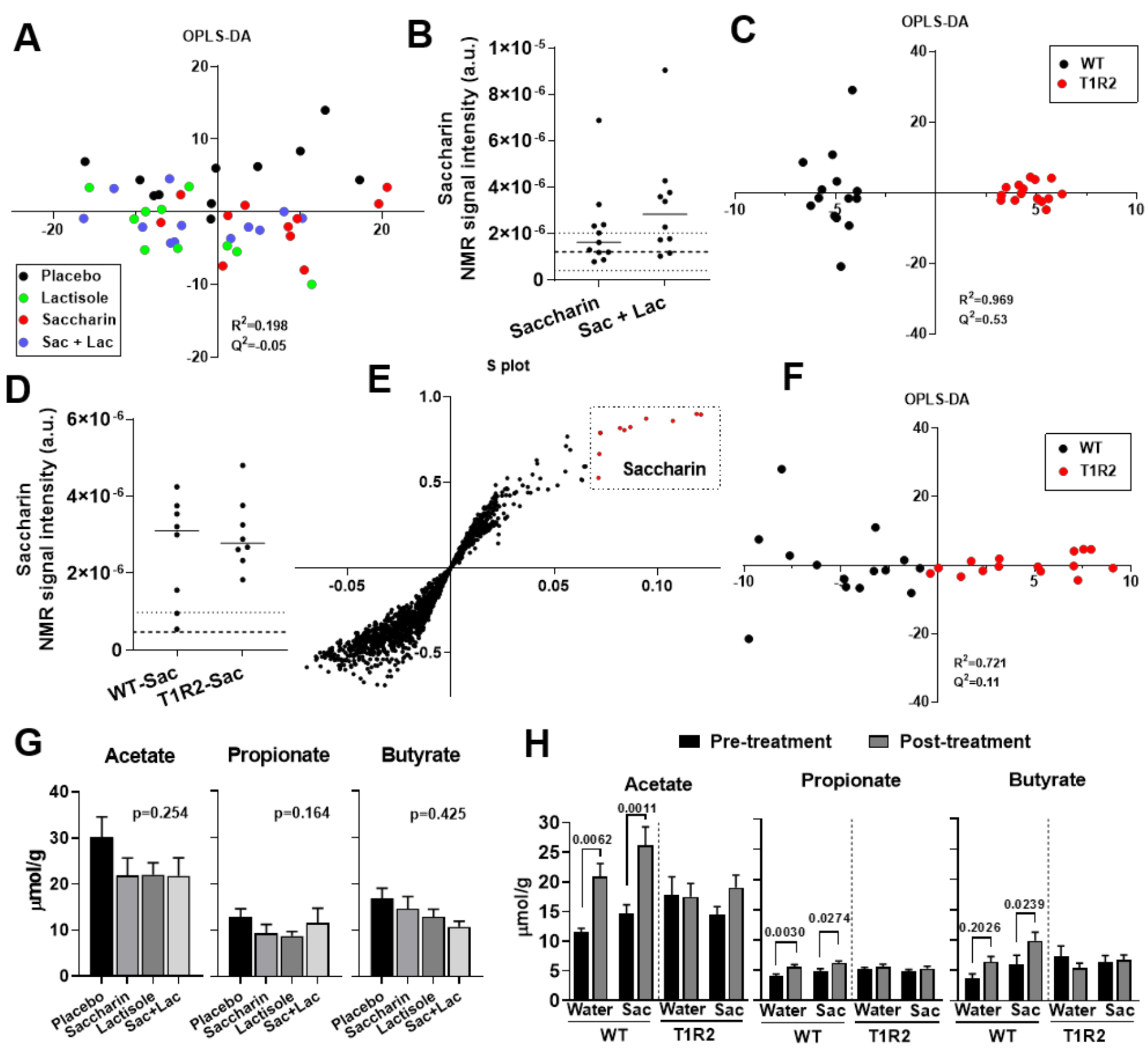


\section{Supplemental methods}

\section{Inclusion and exclusion criteria}

Inclusion criteria: Age 18-45 years apparently healthy; Consumption of less than a can of diet beverage or a spoonful of NCASs weekly (or each equivalent from foods) during the past month; Weight stable $( \pm 3 \mathrm{~kg})$ during the 3 months prior to enrollment; Body Mass Index $(\mathrm{BMI}) \leq 25.0$ $\mathrm{kg} / \mathrm{m}^{2}$.

Exclusion Criteria: Acute or chronic medical conditions or medications that would contraindicate participation in the research testing or could potentially affect metabolic function including, but not limited to: Known coronary artery disease, angina or congestive heart failure; Type 1 or Type 2 Diabetes (A1c $\geq 6.5 \%$ ); Bleeding disorders; Hemoglobin level $<11.5 \mathrm{~g} / \mathrm{dL}$ for women, $<12.0 \mathrm{~g} / \mathrm{dL}$ for men; Acute or chronic infections; Hepatitis and/or cirrhosis (AST or ALT 2.5 times the upper limit of normal); Severe asthma or chronic obstructive pulmonary disease; Renal insufficiency or nephritis (creatinine > $1.6 \mathrm{mg} / \mathrm{dl}$ ); Prior bariatric surgery; Inflammatory bowel disease or malabsorption; Cancer within the last 3 years (except non-melanoma skin cancer or treated cervical carcinoma in situ); Psychiatric disorders or eating disorders; Cushing's disease or syndrome; Untreated or inadequately controlled hypo- or hyperthyroidism (abnormal TSH); Active rheumatoid arthritis or other inflammatory rheumatic disorder; Pregnant or nursing women; Smoking (smoking within the past 3 months); Less than 4 bowel movements per week; Dietary habits exceeding the $10^{\text {th }}-90^{\text {th }}$ percentile of age- and gender-dependent usual macronutrient intake (31); Known hypersensitivity to saccharin, lactisole or any of its excipients. Excluded medications include but are not limited to: Anti-diabetic agents; Oral, injected or chronic topical steroids (inhaled steroids for mild asthma are acceptable); Antibiotic use (within the past 3 months) (32); Other drugs known to affect immune or metabolic function; Orlistat, phenteramine, topirimate or other weight loss or anorectic agents (tricyclic antidepressants, atypical antipsychotics or other psychiatric drugs with effects on body weight). 


\section{Supplemental Fig .1}

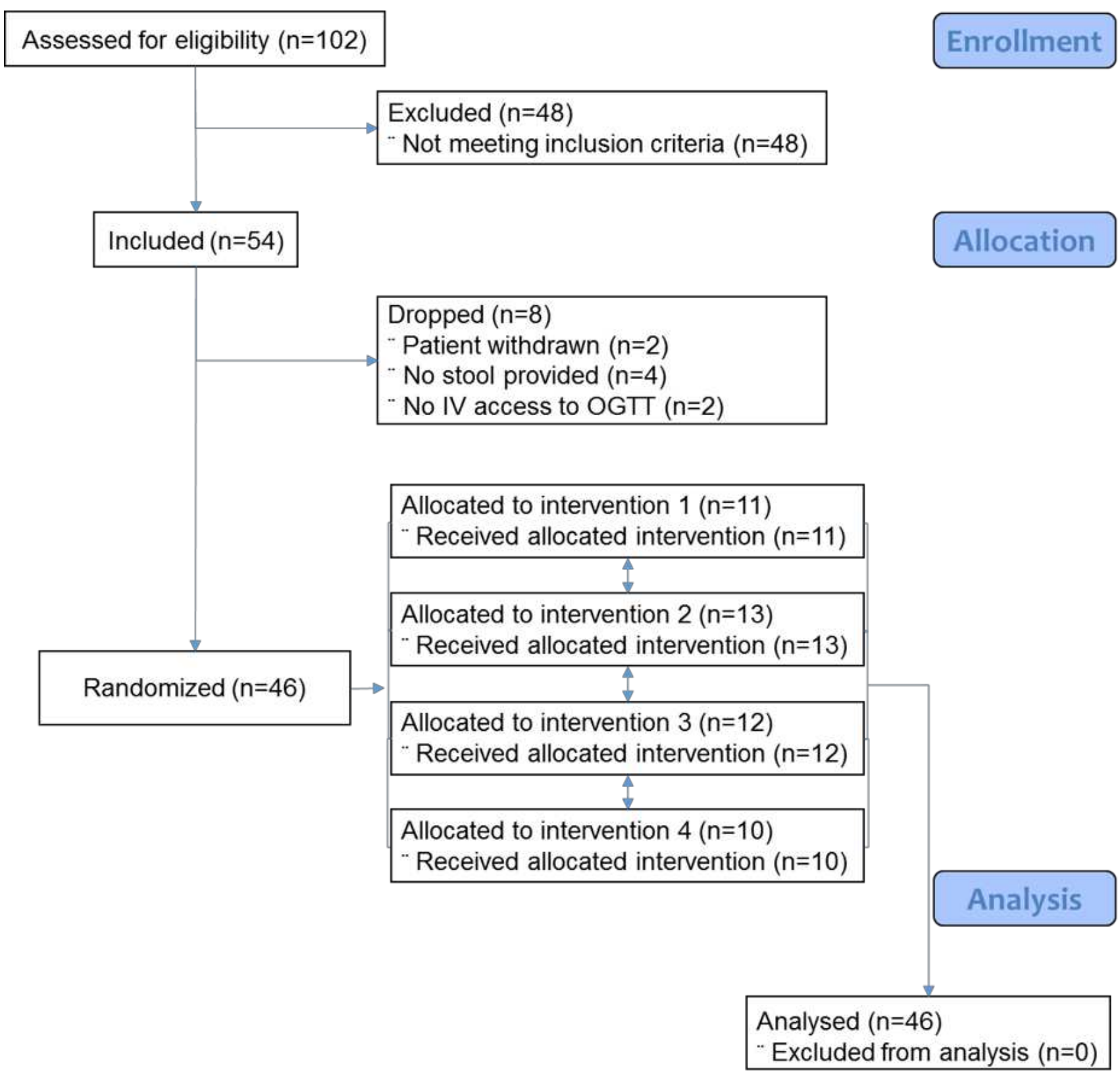

\section{Supplemental figure 1. Participant inclusion flowchart}

A total of 54 subjects out of the 102 screened were allocation among the four experimental groups. Eight non-compliant individuals were removed from the study at various stages. Forty-six randomized subjects completed the interventions. (Intervention 1: Placebo; Intervention 2: Saccharin; Intervention 3: Lactisole; Intervention 4: Saccharin and Lactisole; IV, Intravenous; OGTT, oral glucose tolerance test). 
TABLE S1

Baseline characteristics of participants

\begin{tabular}{lc}
\hline & Value \\
\hline Total (male/female), $\mathrm{n}$ & $46(14 / 32)$ \\
Age, $\mathrm{y}$ & $28.98 \pm 1.29$ \\
Height, $\mathrm{cm}$ & $168 \pm 1.29$ \\
Weight, $\mathrm{kg}$ & $62.95 \pm 1.29$ \\
BMI, $\mathrm{kg} / \mathrm{m} 2$ & $22.26 \pm 0.30$ \\
Glucose, $\mathrm{mg} / \mathrm{dL}$ & $90.33 \pm 1.14$ \\
Triglycerides, mg/dL & $74.82 \pm 5.53$ \\
Total cholesterol, mg/dL & $167.55 \pm 4.56$ \\
HDL, mg/dL & $62.82 \pm 2.07$ \\
LDL, mg/dL & $89.70 \pm 4.09$ \\
Cholesterol:HDL ratio & $2.79 \pm 0.12$ \\
LDL:HDL ratio & $1.53 \pm 0.11$ \\
\hline
\end{tabular}

All values are mean \pm SEM. BMI, body mass index; HDL, high density cholesterol; LDL, low density cholesterol. 


\section{Supplemental Table.2}

\section{TABLE S2}

Participant compliance

Treatment group Length (d)

Placebo

Saccharin

Lactisole

Saccharin + Lactisole

Values are mean \pm SEM

\section{Compliance (\%)}

\begin{tabular}{rr}
$14.2 \pm 0.5$ & $100.4 \pm 3.7$ \\
$14.2 \pm 0.3$ & $100.4 \pm 1.8$ \\
$14.1 \pm 0.3$ & $100.7 \pm 2.2$ \\
$13.7 \pm 0.3$ & $97.4 \pm 2.4$ \\
\hline
\end{tabular}

$100.4 \pm 1.8$

$97.4 \pm 2.4$ 
Placebo
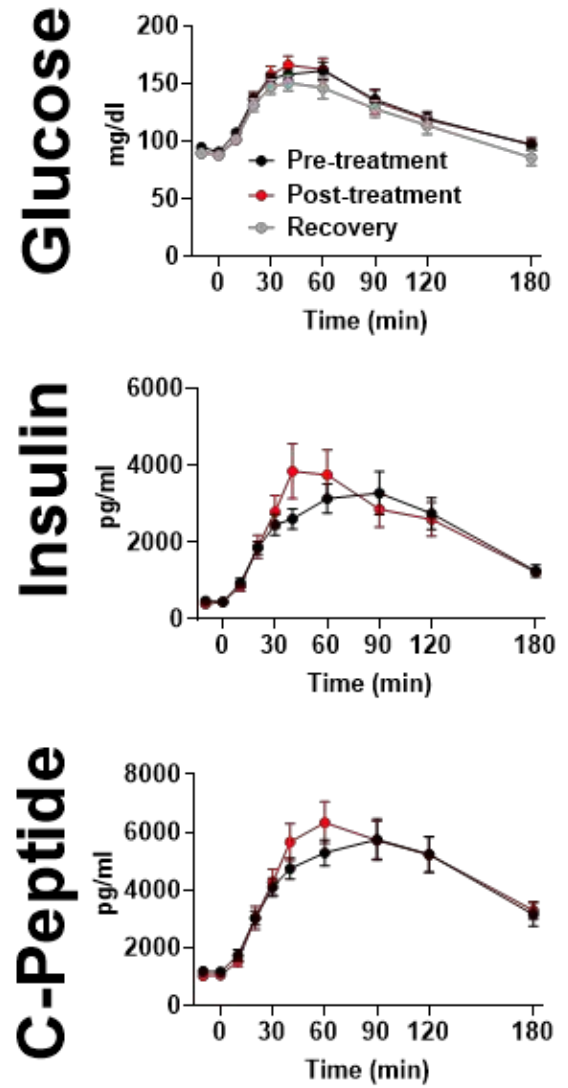

잉
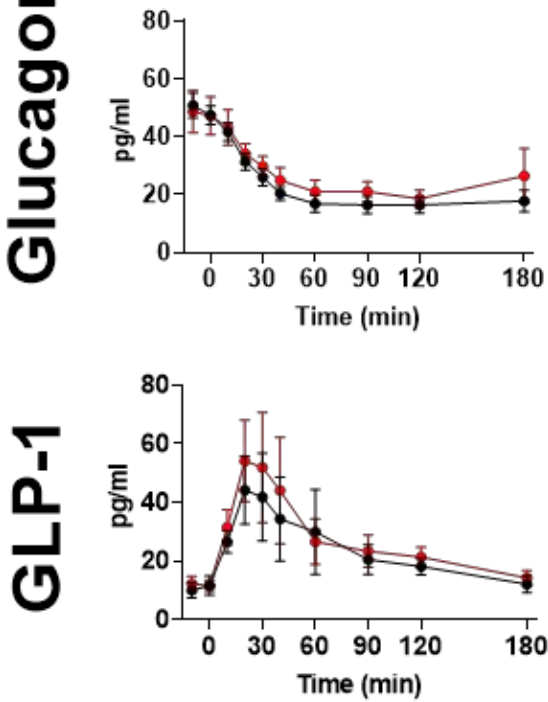

Saccharin
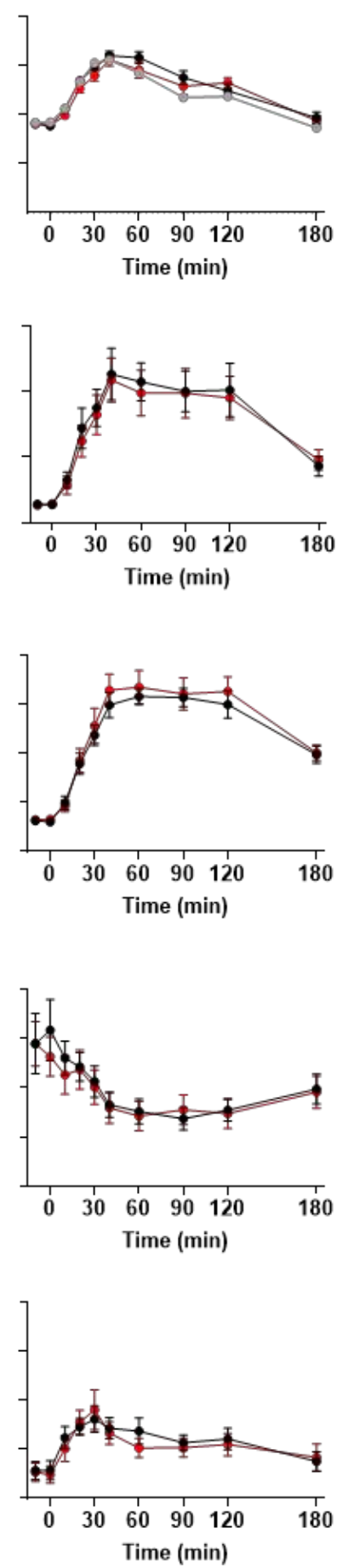
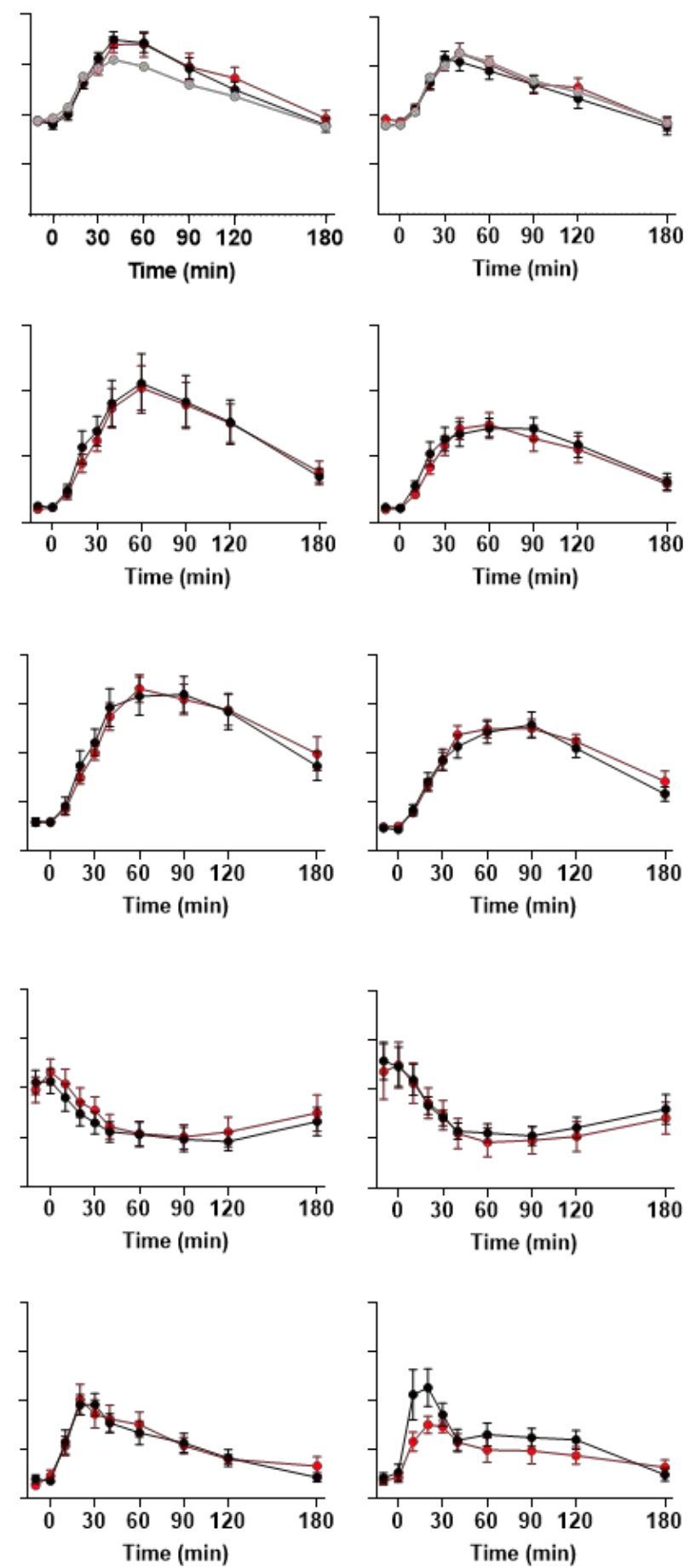

\section{Supplemental figure 2. Longitudinal treatment effects in plasma glucose and hormonal excursions during an OGTT}

Excursions of glucose, insulin, C-peptide, glucagon and GLP-1 during OGTTs before the intervention (pre-treatment), after 2 weeks of intervention (post-treatment), and after the washout period (recovery, only for glucose). Two-way ANOVA repeated measures, $p>0.05$. 
Supplemental Fig. 3
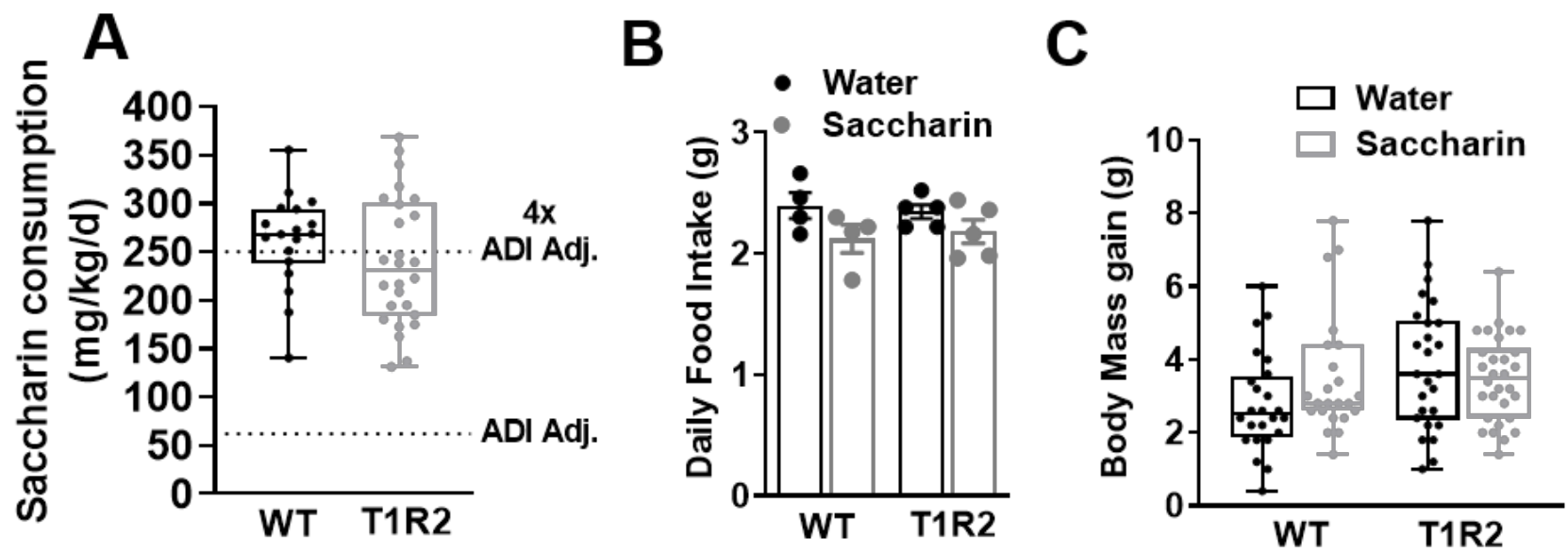

D

WT

T1R2
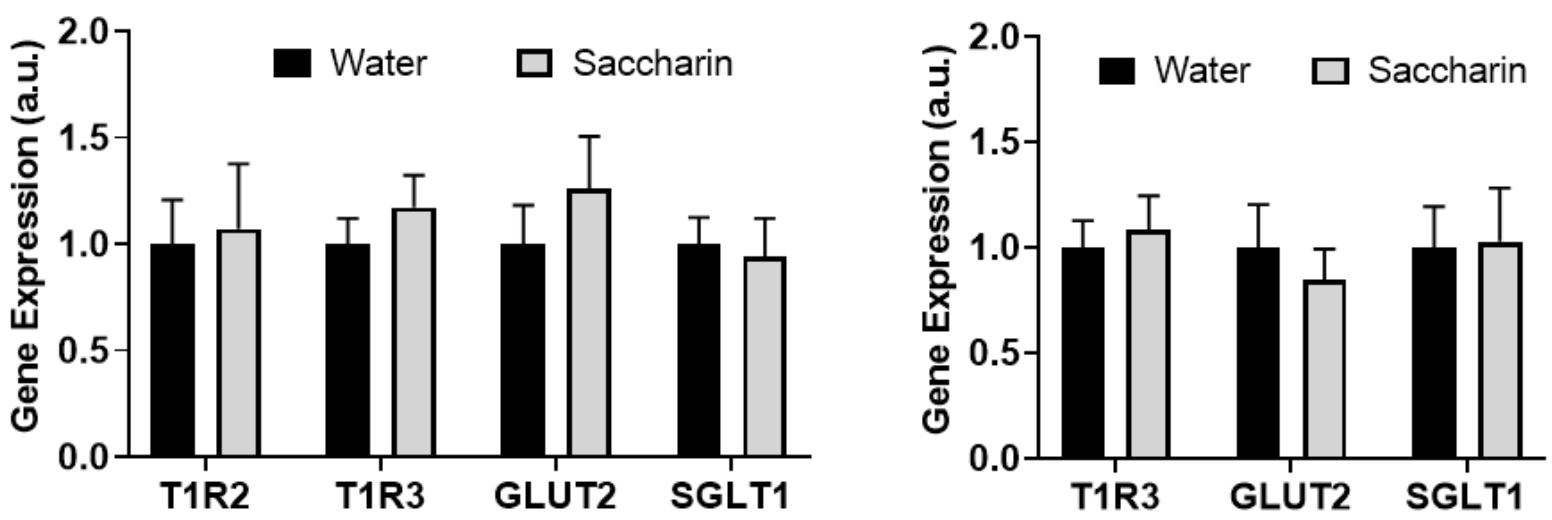

Supplemental figure 3. Treatment compliance and intestinal gene expression in mice Average daily consumption of (A) saccharin or (B) food intake in WT and T1R2 mice. Dotted horizontal lines show saccharin consumption equivalent to human ADI or 4x ADI adjusted for body surface. (C) Body mass gain in response to treatment in mice. (D) Gene expression of T1R2, T1R3, SGLT1 and GLUT2 in jejunal mucosa of mice following treatment. Student's t-test, $p>0.05$ (A and D). Two-way ANOVA, $p>0.5$ (B and C). 
Supplemental Fig. 4

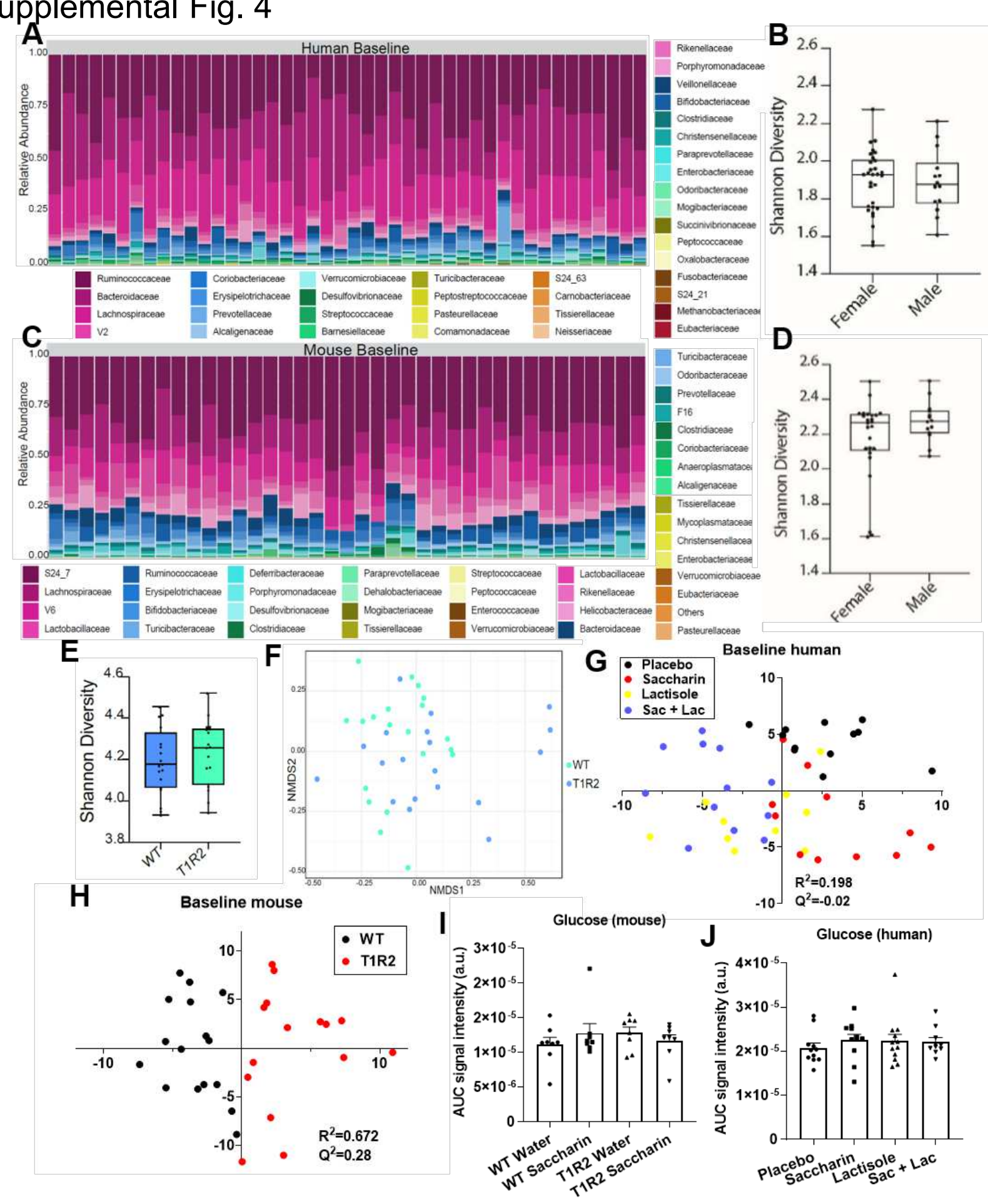

Supplemental figure 4. Assessment of baseline taxonomic abundances and diversity of gut microbiota and metabolomics in humans and mice.

(A and C) Bar chart summary showing relative abundance at the family level before treatment (baeline) in human participants or in WT and T1R2 mice. Each bar represents abundances of one subject. (B and D) Alpha diversity box plot (Shannon diversity metric) showing community richness between gender in human participants (Mann-Whitney $U$ Test; $p=0.77, U=222$ ) or in WT and T1R2 mice (Mann-Whitney U Test; $p=0.987, U=152$ ). (E) Alpha diversity box plot (Shannon diversity metric) showing community richness before treatment between WT and T1R2 mice (Mann-Whitney U Test; $p=0.34, U=142$ ). (F) Nonmetric multidimensional scaling (NMDS) plot showing community similarities between groups before treatment in WT and T1R2 mice $(p<0.19$, NMDS stress $=0.209)$. NMDS ordination was derived from pairwise Bray-Curtis distances and statistical inferences made using PERMANOVA. ( $\mathbf{G}$ and $\mathbf{H}$ ) Differences in human and mouse fecal metabolites before treatment using orthogonal partial least squares discriminant analyses (OPLS-DA). (I and J) Post-treatment glucose presence in human and mouse fecal samples. 
Supplemental Fig. 5

A

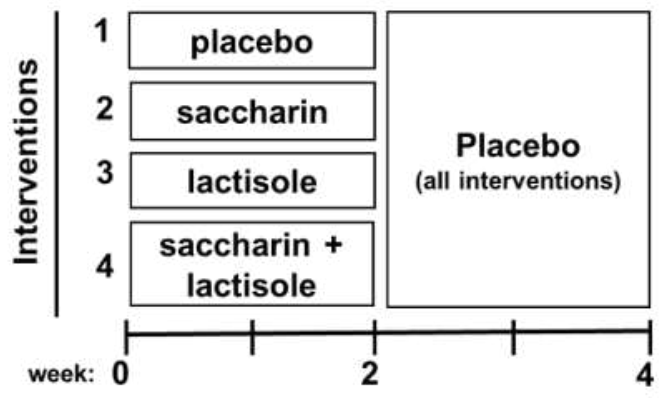

Visit 1

(Pre-intervention)

Visit 3

(Recovery)
B

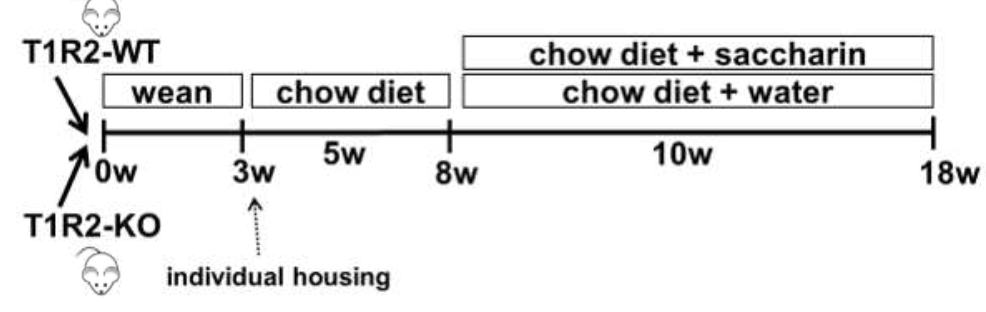

Supplemental figure 5. Experimental design of the human and mouse studies Diagram showing the experimental design of the (A) human and (B) mouse studies. 
Figures
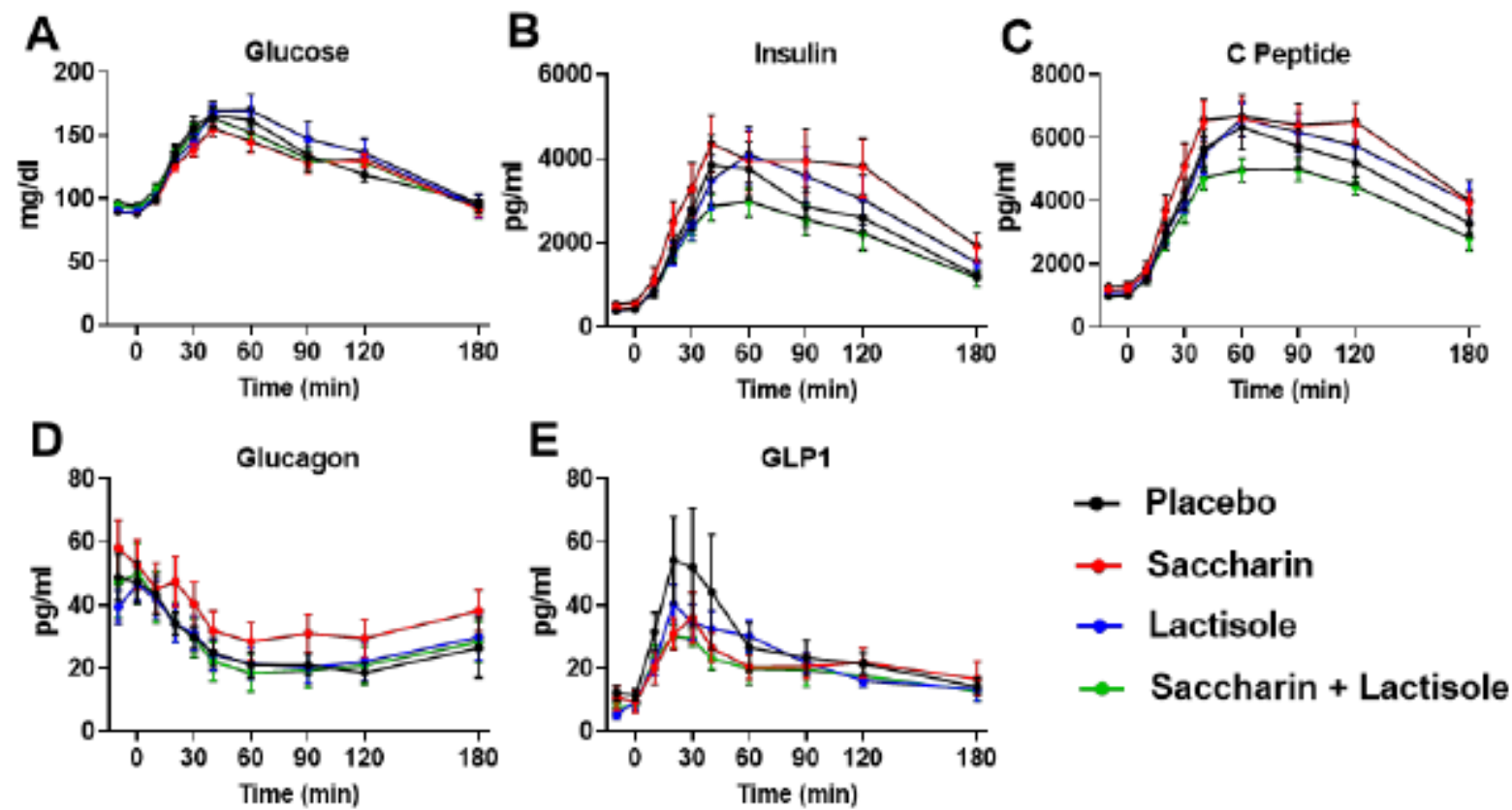

Figure 1

Effects of saccharin and/or lactisole treatment on glucose tolerance in humans Plasma excursions of (A) glucose, (B) insulin, (C) C-peptide, (D) glucagon, and (E) GLP-1 during an oral glucose challenge after 2 weeks of treatment. Two-way ANOVA repeated measures ( $p>0.05)$. $N=10-13$. 

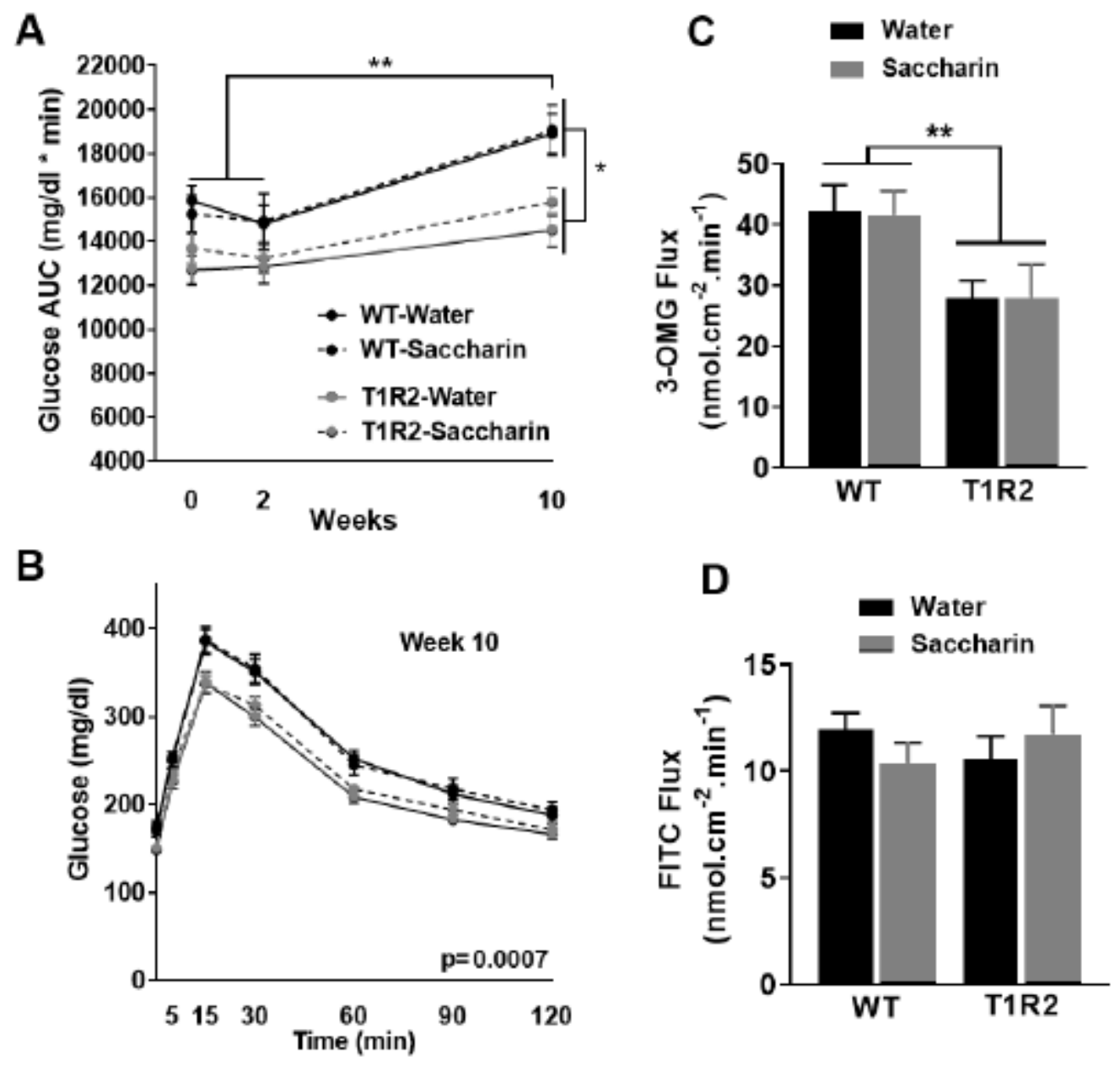

Figure 2

Effects of saccharin treatment on glucose homeostasis in mice (A) Glucose responses during an i.g.GTT expressed as area under curve (AUC) before ( 0 weeks), 2 and 10 weeks after saccharin treatment. Twoway ANOVA main effect; ${ }^{*} p<0.05$, WT 10-week vs. T1R2 10-week, ${ }^{* *} p<0.01$ WT 0-week vs. WT 10-week). (B) Glucose excursions during an i.g.GTT in response to 10 weeks of saccharin treatment (2-way ANOVA repeated measures, $p=0.0007$ ). (C) Ex vivo glucose flux using 3-0-methy-glucose (3-OMG) in intact mouse intestines following 10 weeks of saccharin treatment. Two-way ANOVA, ${ }^{*} p<0.01$. (D) Ex vivo intestinal permeability assessed by FITC-dextran (4kDa) flux in intact mouse intestines following 10 weeks of saccharin treatment. Two-way ANOVA, $p>0.05$. $N=23-28$ for in vivo studies, $n=6-11$ for ex vivo studies. 


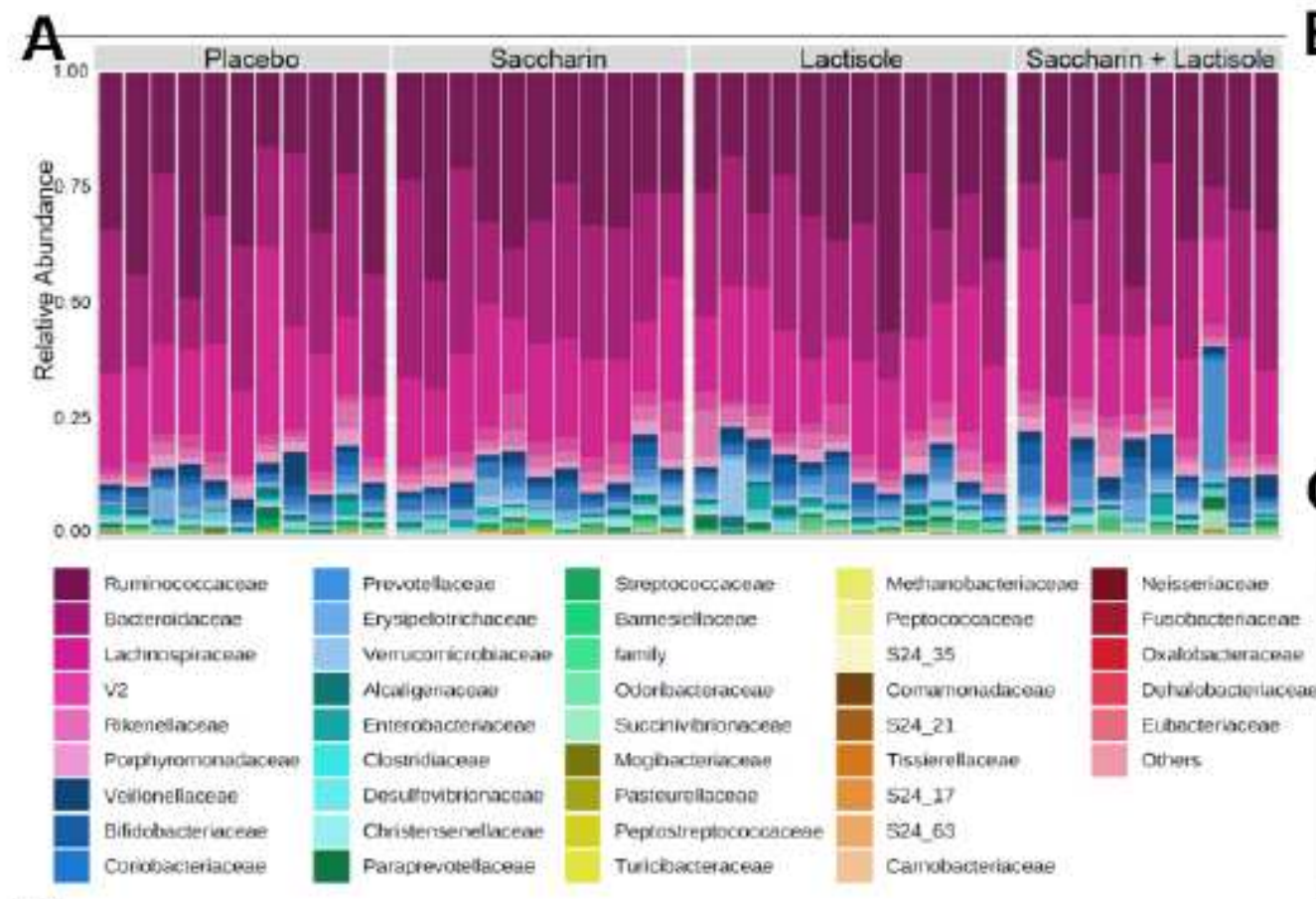

B
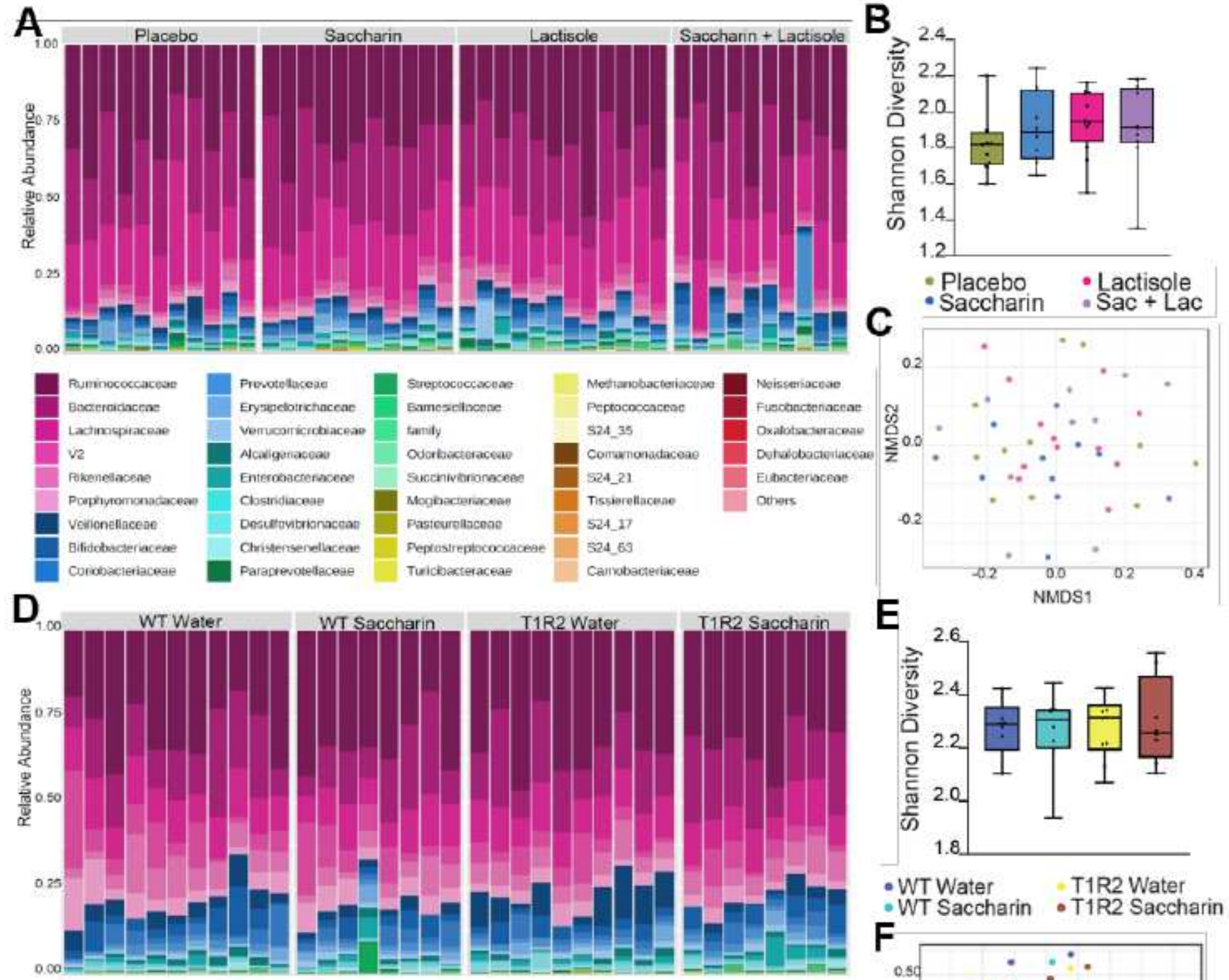

\begin{tabular}{|c|c|c|c|c|}
\hline $524 \ldots$ & Turichacteraceae & Desullowbricntecese & Paraprevotedocese. & Staprifolococcaceat \\
\hline Lacthospracese & Odonibacteraceso & Caricbacteriacease & Tissierellacease & Eubacietiacease \\
\hline vo & B fidobocteniacenes & Planococcaceare & Ventucorricrobiaceave & Pasteuretaceale \\
\hline Lactohecillsosese & Prevotelincene & Anseroptasmatnceste & Christensenelacene & Peptostreptococcaceas \\
\hline Fokernelaceas & F16 & Debalobacteriaceas & Peptococcaces: & Besilacene \\
\hline Bacteicidaceas & Eryspelouichaceas & Aral genaceat & Enterobactetiaceae & oviers \\
\hline Heicobacteraceae & Poiptyromonadsceae & Costrinticeac & Emetococcaceas & \\
\hline Ruminococcacese & Defernoacteracosae & Mogbacteriaceos & stregrococcacese & \\
\hline
\end{tabular}

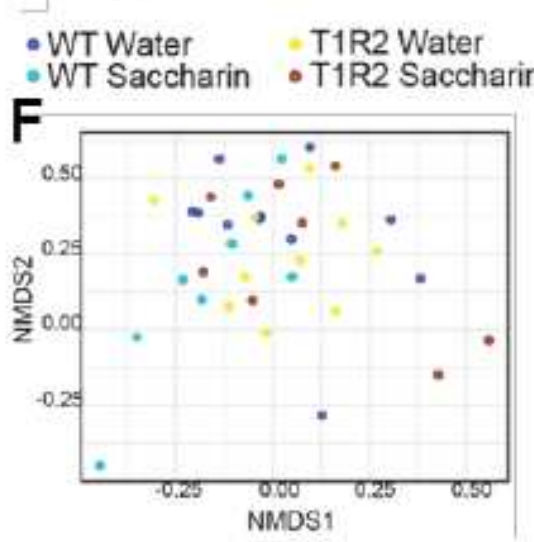

\section{Figure 3}

Taxonomic abundances and diversity of gut microbiota in response to treatments in humans and mice (A and D) Bar chart summary showing relative abundance at the family level post-treatment inhuman participants or in WT and T1R2 mice. Each bar represents abundances of one subject. (B and E) Alpha diversity box plot (Shannon diversity metric) showing community richness between groups posttreatment in human participants (Mann-Whitney U Test; $p=0.156, U=5.22$ ) or in WT and T1R2 mice (Mann-Whitney U Test; $p=0.987, \mathrm{U}=152$ ). (C and F) Nonmetric multidimensional scaling (NMDS) plot showing community similarities between groups post-treatment in human participants $(p<0.999$, NMDS 
stress $=0.2274)$ or WT and T1R2 mice $(p<0.111$, NMDS stress $=0.209)$. NMDS ordination was derived from pairwise Bray-Curtis distances and statistical inferences made using PERMANOVA. N=11-13 for human studies, $n=8-11$ for mouse studies.
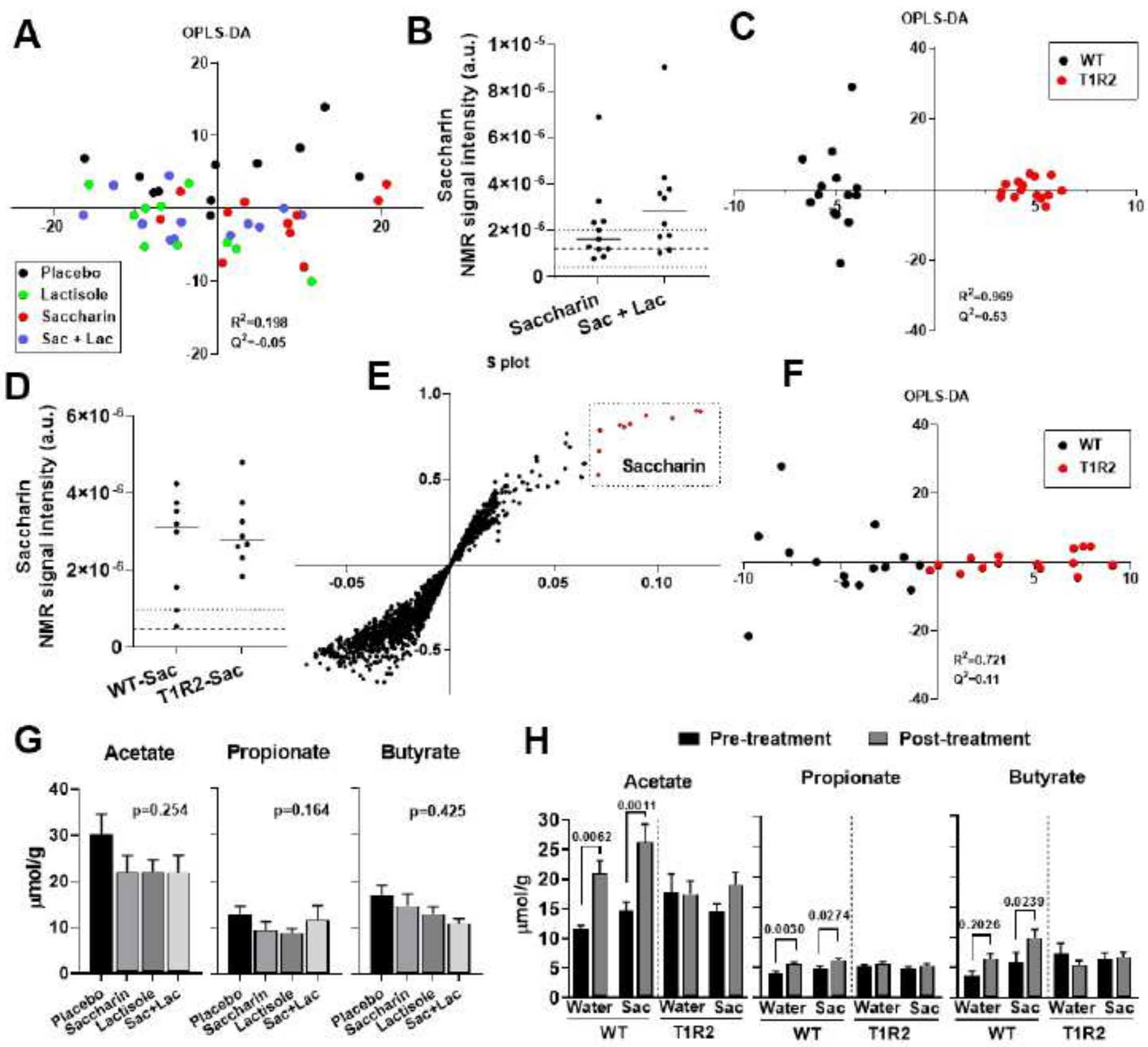

Figure 4

Fecal metabolomics in response to treatments in humans and mice (A) Differences in human fecal metabolites between treatment groups using orthogonal partial least squares discriminant analyses (OPLS-DA). (B) Post-treatment saccharin presence in human fecal samples. Dashed lines represent average noise \pm SD. (C) Differences in WT andT1R2 fecal metabolites following saccharin treatment using OPLS-DA. (D) Post-treatment saccharin presence in mouse fecal samples. Dashed lines represent 
average noise \pm SD. (E) Metabolite distribution (S-plot) in fecal mouse samples. Metabolites attributed to saccharin shown in red. (F) Differences in WT and T1R2 fecal metabolites following saccharin treatment using OPLS-DA after removal of saccharin signals. (G) Assessment of short chain fatty acids (SCFA) following treatments in human samples. One-way ANCOVA baseline as covariate. $(H)$ SCFA in mouse feces before (pre) and after (post) treatment. Two-way ANOVA repeated measures with post-hoc. N=11-13 for human studies, $\mathrm{n}=8$ for mouse studies. 